\title{
THE CONNECTIONS OF THE MIDDLE TEMPORAL VISUAL AREA (MT) AND THEIR RELATIONSHIP TO A CORTICAL HIERARCHY IN THE MACAQUE MONKEY ${ }^{1}$
}

\author{
JOHN H. R. MAUNSELL ${ }^{2}$ AND DAVID C. VAN ESSEN \\ Division of Biology 216-76, California Institute of Technology, Pasadena, California 91125
}

Received January 5, 1983; Revised April 25, 1983; Accepted June 6, 1983

\begin{abstract}
The cortical and subcortical connections of the middle temporal visual area (MT) of the macaque monkey were investigated using combined injections of $\left[{ }^{3} \mathrm{H}\right]$ proline and horseradish peroxidase within MT. Cortical connections were assigned to specific visual areas on the basis of their relationship to the pattern of interhemispheric connections, revealed by staining for degeneration following callosal transection. MT was shown to be reciprocally connected with many topographically organized cortical visual areas, including V1, V2, V3, and V4. These pathways link regions representing corresponding portions of the visual field in the different areas. In addition, MT has reciprocal connections with two previously unidentified cortical areas, which we have designated the medial superior temporal area (MST) and the ventral intraparietal area (VIP).

The laminar distribution of terminals and cell bodies in cortical areas connected with MT follows a consistent pattern. In areas V1, V2, and V3, the projections to MT arise largely or exclusively from cells in supragranular layers, and the reciprocal connections from MT terminate mainly in supragranular and infragranular layers. In contrast, the projections to MST and VIP terminate mainly in layer IV, and the reciprocal pathways originate from cells in both superficial and deep layers. On the basis of this pattern, each connection can be designated as forward or feedback in nature, and a hierarchical arrangement of visual areas can be determined. In this hierarchy, MT is at a higher level than V1, V2, and V3, and at a lower level than MST and VIP.

Subcortical projections were seen from MT to the claustrum, the putamen, the caudate nucleus, the inferior and lateral subdivisions of the pulvinar complex, the ventral lateral geniculate nucleus, the reticular nucleus of the thalamus, the superior colliculus, and the pontine nuclei.
\end{abstract}

The finding of a large number of intricately connected cortical and subcortical visual areas in higher mammals has made it clear that there is a very complex network involved in the processing of visual information. In the macaque monkey, anatomical tracing techniques have been instrumental in demonstrating that there are at least 10 visual areas in the cerebral cortex (see Van Essen et al., 1982) and in suggesting how information is distributed among them (see Weller and Kaas, 1981).

\footnotetext{
${ }^{1}$ We thank C. Shotwell for histological work and preparation of figures, and C. Hochenedel and C. Oto for typing the manuscript. We also thank Drs. G. Standage and L. Benevento for providing access to their manuscript in press. This work was supported by National Research Award S T32 GM 07737 from the National Institute of General Medical Services, by Research Grant R01 EY 02091 from the National Eye Institute, and by the Pew Memorial Trust.

${ }^{2}$ To whom reprint requests should be sent, at his present address: Department of Psychology, Building E25-634, Massachusetts Institute of Technology, Cambridge, MA 02139.
}

This report concerns the anatomical connections of one of the extrastriate visual areas in the macaque, the middle temporal area (MT). MT is a small area on the posterior bank of the superior temporal sulcus which receives a direct input from V1 - striate cortex (Cragg, 1969; Zeki, 1969). It has a high percentage of neurons which are selective for the direction, speed, and binocular disparity of moving stimuli, suggesting it is important in the analysis of visual motion (Zeki, 1974a, b; Maunsell and Van Essen, 1983a, b). It is obviously of interest to know exactly what inputs it gets, which centers receive its output, and whether these targets are also specialized for motion analysis. Such information is also of interest from a comparative viewpoint, as the connections of MT have been studied extensively in several other primate species (Spatz and Tigges, 1972, 1973; Tigges et al., 1981, 1982; Wall et al., 1982).

In the present study the anatomical connections of MT were examined using anterograde and retrograde 
tracers. We found that MT has reciprocal connections with a large number of identifiable cortical areas and also extensive connections with subcortical centers. Two of the cortical targets of MT are areas previously unidentified in the macaque. Although there are numerous pathways leading to and from $\mathrm{MT}$, considerable order exists in the system of connections, and it is possible to delineate a clear hierarchy of cortical visual areas based on objective anatomical criteria. Finally, by analyzing the fine pattern of connections, we have obtained intriguing clues pertaining to the functional heterogeneity within individual cortical areas. An abstract of some of this work has previously been published (Maunsell and Van Essen, 1982).

\section{Materials and Methods}

Anatomical tracers were injected into MT in the right hemispheres of three Macuca fascicularis which weighed between 3.1 and $3.7 \mathrm{~kg}$. Because MT is small and there is considerable individual variability in its position within the superior temporal sulcus (Van Essen et al., 1981), it was necessary to establish the location of MT in each animal with physiological recordings before the injection. All of the animals had been used for semichronic recordings from MT, the results of which have been reported elsewhere (Maunsell and Van Essen, $1983 \mathrm{a}, \mathrm{b})$. In the course of these recordings the extent and topography of MT were largely established in each of the experimental hemispheres, and this mapping was used as a guide for determining where to make the injections. With this technique it was possible to make injections which were entirely confined to MT and which involved the representation of a particular part of the visual field.

Injections were made using a $1-\mu \mathrm{l}$ syringe (Hamilton) containing $25 \mu \mathrm{Ci}$ of $\left[{ }^{3} \mathrm{H}\right]$ proline in $150 \mathrm{nl}$ of a $20 \%$ solution of horseradish peroxidase (HRP; Boehringer Mannheim). To reduce damage by the syringe, the original barrel was replaced with a 30 gauge syringe needle (300- $\mu \mathrm{m}$ outside diameter). Leakage of tracers was minimized by loading $30 \mathrm{nl}$ of a $30 \%$ sucrose solution into the syringe both in front of and behind the tracers. The approach to MT was horizontal, running through striate cortex and the lunate sulcus to reach MT from the white matter. For two injections the syringe needle was coated with a thin layer of insulating varnish everywhere except at the tip. Multi-unit recordings were made to confirm the depth of MT and the visual field representation before the injection. For the other injection, a microelectrode penetration to identify M'T was made immediately before the syringe was inserted. Injections were made at a rate of about $120 \mathrm{nl} / \mathrm{hr}$, and there was a 15 -min pause between the end of the injection and retracting the syringe. For the two injections made with the recording syringe, the animal was paralyzed and respired with nitrous oxide. For the other injection, the animal was sedated intramuscularly with ketamine.

The splenium of the corpus callosum (posterior $1 \mathrm{~cm}$ ) was transected to provide an anatomical marker for the boundaries of some of the extrastriate visual areas (Zeki, 1970; Van Essen et al., 1982). The callosum was cut 3 to 4 days after the tracers were injected to allow time for label to be transported to the opposite cerebral hemisphere. This surgery was performed under aseptic conditions and with sodium thiopental anesthesia.

Four or five days after the corpus callosum was cut, an acute recording session was made to map receptive fields in MT and the surrounding cortex in the left (uninjected) hemisphere. The recording techniques used for this session have been described in detail elsewhere (Van Essen et al., 1981). At the end of this session, which lasted 1 to 2 days, the animal was deeply anesthetized with sodium pentobarbitol and perfused through the heart with a phosphate buffer rinse $(0.1 \mathrm{M}, \mathrm{pH} 7.4)$ followed by a series of buffered $4 \%$ formaldehyde solutions containing $0 \%, 10 \%$, and $20 \%$ sucrose. The brain was then removed, photographed, blocked, and allowed to equilibrate with phosphate buffer containing $30 \%$ sucrose.

The blocks were sectioned at $31 \mu \mathrm{m}$. Sections every $0.25 \mathrm{~mm}(1 \mathrm{in} 8)$ were reacted for HRP using a modification of the tetramethyl benzidine method (Mesulam, 1978) in which the incubation and reaction were carried

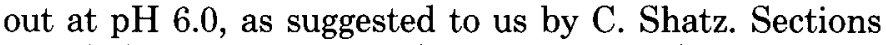
were lightly counterstained with neutral red before scanning for labeled cells with brightfield microscopy. Two other series of sections at $0.25-\mathrm{mm}$ spacing were stored in buffered $4 \%$ formaldehyde for 2 to 12 weeks and then processed for autoradiography (Cowan et al., 1972) and fiber degeneration (Wiitanen, 1969). Autoradiographic sections were exposed for 3 to 6 weeks. A final series of sections was stained for myelin (Gallyas, 1979) and was used to determine the borders of MT (Van Essen et al., 1981). All histological data were scored on $\times 8$ photographic enlargements of the individual sections and subsequently transferred to two-dimensional maps of the cortex for analysis (Van Essen and Maunsell, 1980).

The survival time and fixation procedures for these experiments were chosen to optimize conditions for autoradiography and degeneration staining and to allow time for transport to the opposite hemisphere before the callosum was cut. These conditions were certainly less than ideal for HRP reactivity, however, and there were fewer IIRP-labeled cells than we have seen for smaller injections in other experiments with shorter survival times and different fixation procedures (unpublished results). Nonetheless, a reasonable degree of retrograde labeling was seen in many different regions, and we are confident that the HRP injections were adequate for identifying the major cortical inputs to MT.

\section{Results}

Location of injection sites. The locations of the three $\left[{ }^{3} \mathrm{H}\right]$ proline injections in MT are shown in photomicrographs of sections in Figure 1. Those for injections 1 and 2 were cut in the parasagittal plane, and that of injection 3 was cut horizontally. Each section is within $0.5 \mathrm{~mm}$ of the center of the injection. 'The borders of MT could be determined to an accuracy of about $1 \mathrm{~mm}$ on nearby sections stained for myelin; the range of uncertainty for the location of each border is indicated by a black line below the cortex in the photographs of Figure 1.

Injections 1 and 2 were centered near layer IV and were restricted to the gray matter of MT, there being no 

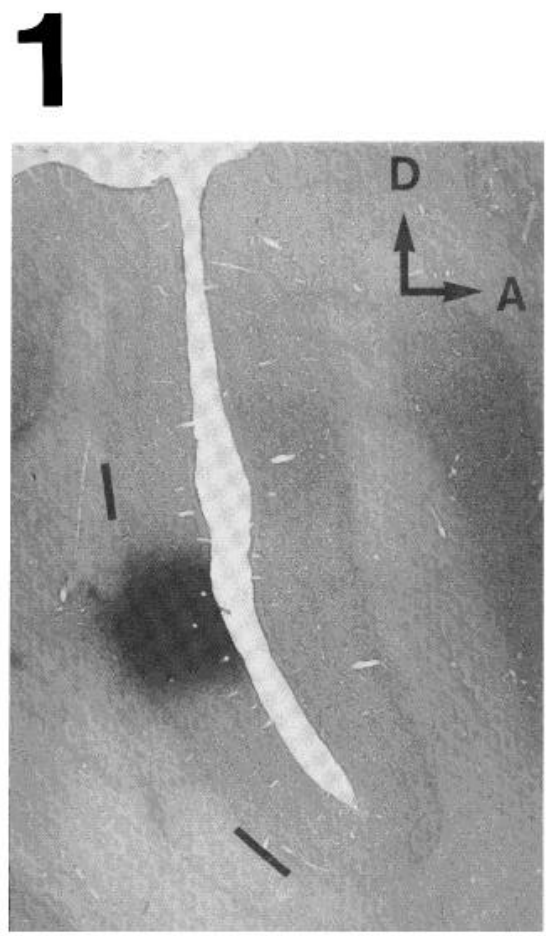
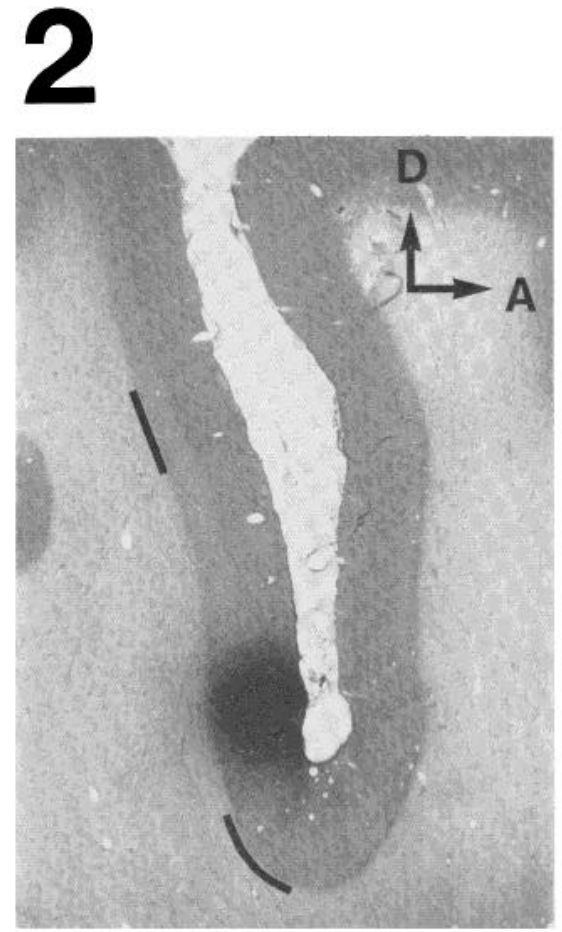
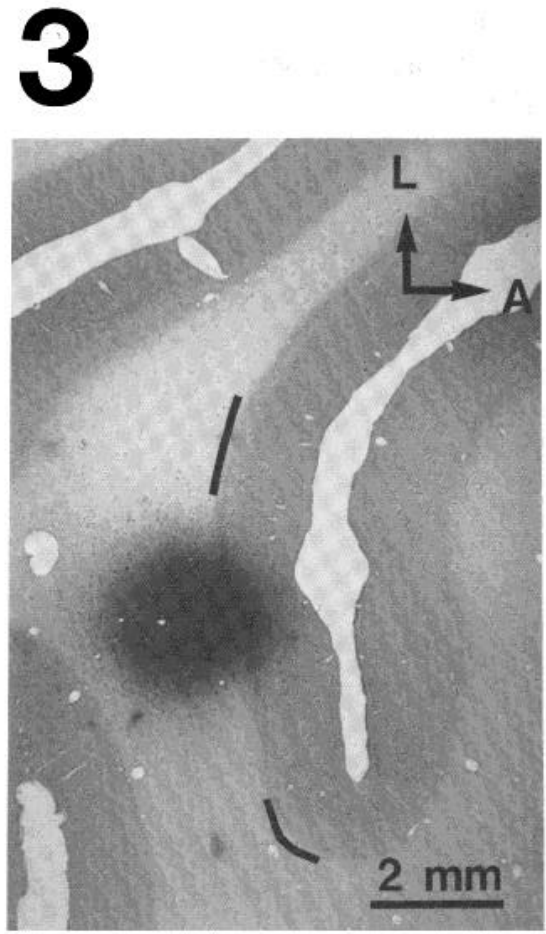

Figure 1. Photomicrographs of the $\left[{ }^{3} \mathrm{H}\right]$ proline injections of MT. These sections were taken from within $0.5 \mathrm{~mm}$ of the center of each injection, processed for autoradiography, counterstained for Nissl substance, and photographed under brightfield illumination. The plane of section for injections 1 and 2 was parasagittal, that for injection 3 was horizontal. The black lines below the cortex indicate the range of uncertainty in assigning the borders of MT on nearby sections stained for myelin. Injection 3 was centered in white matter below MT but extended well into the infragranular layers. The HRP data from this injection were discounted. The sections for injections 1 and 2 were exposed for 3 weeks, that for injection 3 was exposed for 6 weeks. Survival time after the injection was 10 days for injection 1 and 9 days for the others.

significant leakage of tracers along the syringe tracks. Injection 3 was centered in white matter below MT, but it extended well into the infragranular layers. Unlike the others, this injection resulted in HRP-labeled cells over most of the prelunate gyrus, presumably as a result of uptake by damaged axons in the white matter around the injection site (Grob et al., 1982). For this reason the HRP data for this animal were discounted. Fortunately, the autoradiography showed a more restricted distribution of transported label. This indicates that substantial proline uptake did not occur in white matter, just as has been found in control experiments under comparable conditions in our laboratory (unpublished results). For the two successful HRP cases, the apparent size of the injection site was about 1 to $1.5 \mathrm{~mm}$ in diameter, slightly smaller than for the $\left[{ }^{3} \mathrm{H}\right]$ proline injections. Even though our processing for HRP reactivity was less than optimal (see "Materials and Methods"), it is very unlikely that we substantially underestimated the effective size of the HRP injection, because the distribution of retrogradely labeled cells was slightly more restricted than that for anterograde transport (see Figs. 3 and 4, below).

Figure $2 A$ contains two-dimensional maps of $\mathrm{MT}$ in which the locations and approximate sizes of the $\left[{ }^{3} \mathrm{H}\right]$ proline injection sites have been marked. These maps include only cortex in and immediately surrounding MT; they were made by aligning adjacent contours of layer IV taken from histological sections (Van Essen and
Maunsell, 1980). The representation is as though one were looking at part of the superior temporal sulcus after it had been opened by unfolding. The dashed line in Figure $2 A$ denotes the fundus of the sulcus, and the posterior bank is to the left. The borders of MT are marked by drawing smooth lines approximately midway through the regions of uncertainty for the borders on each section. All of the injections were in the dorsal half of MT, where the more peripheral parts of the visual field are represented (Van Essen et al., 1981).

The receptive fields of cells at or near each of the injection sites are drawn in Figure $2 B$. The receptive fields for injections 1 and 2 were plotted at the time of the injections using a syringe which had been modified for multi-unit recording. Both injections were in the representation of the inferior quadrant: injection 1 was at an eccentricity of 6 to $7^{\circ}$ and close to the representation of the vertical meridian; injection 2 was more peripheral ( $35^{\circ}$ eccentricity) and away from both the horizontal and vertical meridians. Recordings were not made at the time of injection 3 , but the approximate location can be confidently assigned to the region of the horizontal meridian representation at about $10^{\circ}$ to $15^{\circ}$ eccentricity, based on previous recordings made in the vicinity of the injection site (see figure legend) and also on the distribution of projections to V1 and V2 (see below). It is evident that, within MT, receptive field locations are poorly correlated with the positions of injection sites. 
This is consistent with the irregular topography and individual variability previously found in MT (Van Essen et al., 1981).

\section{Cortical connections}

Assignment of cortical connections. All three experiments revealed an extensive, complex pattern of connections with at least six cortical areas, and one of the injections indicated that there are connections with one or two additional areas. Most of these connections can be assigned with confidence to specific visual areas. This section will deal with the identification of the cortical areas with which MT is connected and with the laminar distribution of these connections. A subsequent section will address the topographic order of these connections, the uniformity or patchiness of label distribution in different areas, and the intrinsic connections of MT.

The distribution of connections in relation to established visual areas is best appreciated by considering one

A
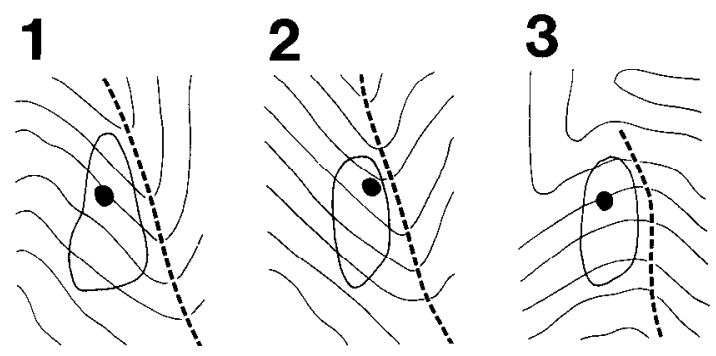

$4 \mathrm{~mm}$
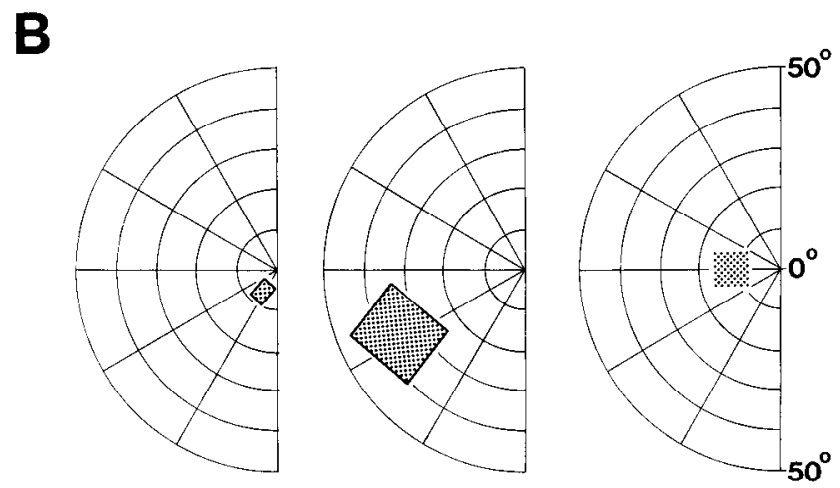

Figure 2. A, Two-dimensional maps of the injections. The location and size of the injections are shown in two-dimensional maps of the cortex in and around MT in the superior temporal sulcus. The perspective is as if one is looking down on the corlex after the sulcus has been unfolded. The dashed line is the fundus of the sulcus, and the posterior bank is to its left. The border of MT is drawn as a solid line. Each injection was confined to the cortex within MT and involved a small fraction of its total area. $B$, The locations of the receptive fields of cells at or near the injection sites. The receptive fields for injections 1 and 2 were plotted at the time of the injection using a syringe modified for recording. Although no recording was made at the time of injection 3 , the position of the receptive field representation at this site can be assigned with confidence based on prior recordings (four penetrations within $2 \mathrm{~mm}$ of the injection site) and on the location of label in visual areas with well defined topographies. case in detail. The complete pattern of cortical connections for injection 1 is shown on separate copies of a twodimensional cortical map in Figure $3 A\left(\left[{ }^{3} \mathrm{H}\right]\right.$ proline $)$ and Figure $3 B$ (HRP). The mapping unfolds the convoluted visual cortex into a flat sheet with relatively little areal distortion (Van Essen and Maunsell, 1980). The obvious advantage of this type of representation is that in a single view one can see the entire pattern of projections, as well as the size, shape, and local relationships of labeled regions, whereas these features are difficult to discern from the locations of labeled zones on a series of sections. The map is similar to those in Figure 2, except that the representation is expanded to include all of the visual cortex. More anterior regions of the cortex are represented mainly to the right, and dorsal and ventral regions are on upper and lower parts of the map, respectively. All of the striate cortex is represented by the elliptical region on the left; it has been separated from extrastriate cortex by a discontinuity along its margins, introduced to reduce distortions. A dashed line in Figure 3 marks the fundus of each sulcus. MT, whose borders have been outlined, lies roughly in the center of the extrastriate portion of the map, near the fundus of the superior temporal sulcus. In Figure $3 A$ anterogradely transported label is indicated by hatching, and in Figure $3 B$, the locations of HRP-labeled cells are marked with dots.

The connections revealed by this injection were mostly in the dorsal part of the hemisphere (upper portion of the cortical map). This is not surprising, given that the injection was within the representation of the inferior quadrant, and that inferior fields are represented predominantly in the dorsal part of the occipital lobe. Connections with many distinct regions can be seen for both the HRP and the proline injections. The patterns for the two tracers are remarkably similar, and for convenience, corresponding connections have been numbered in identical fashion. The correspondence of anterograde and retrograde transport implies that the cortical pathways to and from MT are reciprocal not only in terms of which areas are connected, but also in the fine patterns within individual areas. The only substantial difference is that no retrodradely labeled cells are visible in some of the regions with weak anterograde label. As already noted, this is most likely attributable to the greater effectiveness of the $\left[{ }^{3} \mathrm{H}\right]$ proline injections under the particular conditions of these experiments.

The connections with V1 and V2 can be identified easily on the basis of their relationship to sharp architectonic transition at the V1/V2 border. Except for V1 and MT, through, cortical architectonics has been of limited use for identifying visual areas in the occipital lobe, and it was important to have additional criteria for the location of particular areas. The principal marker we used was the pattern of callosal inputs, which are preferentially distributed along regions of vertical meridian representation and which provide a useful guide for determining the borders of a number of topographically organized visual areas, including V3, VP, V3A, and V4 (Van Essen and Zeki, 1978; Van Essen et al., 1982). Callosal inputs were demonstrated by staining for degen- 
A
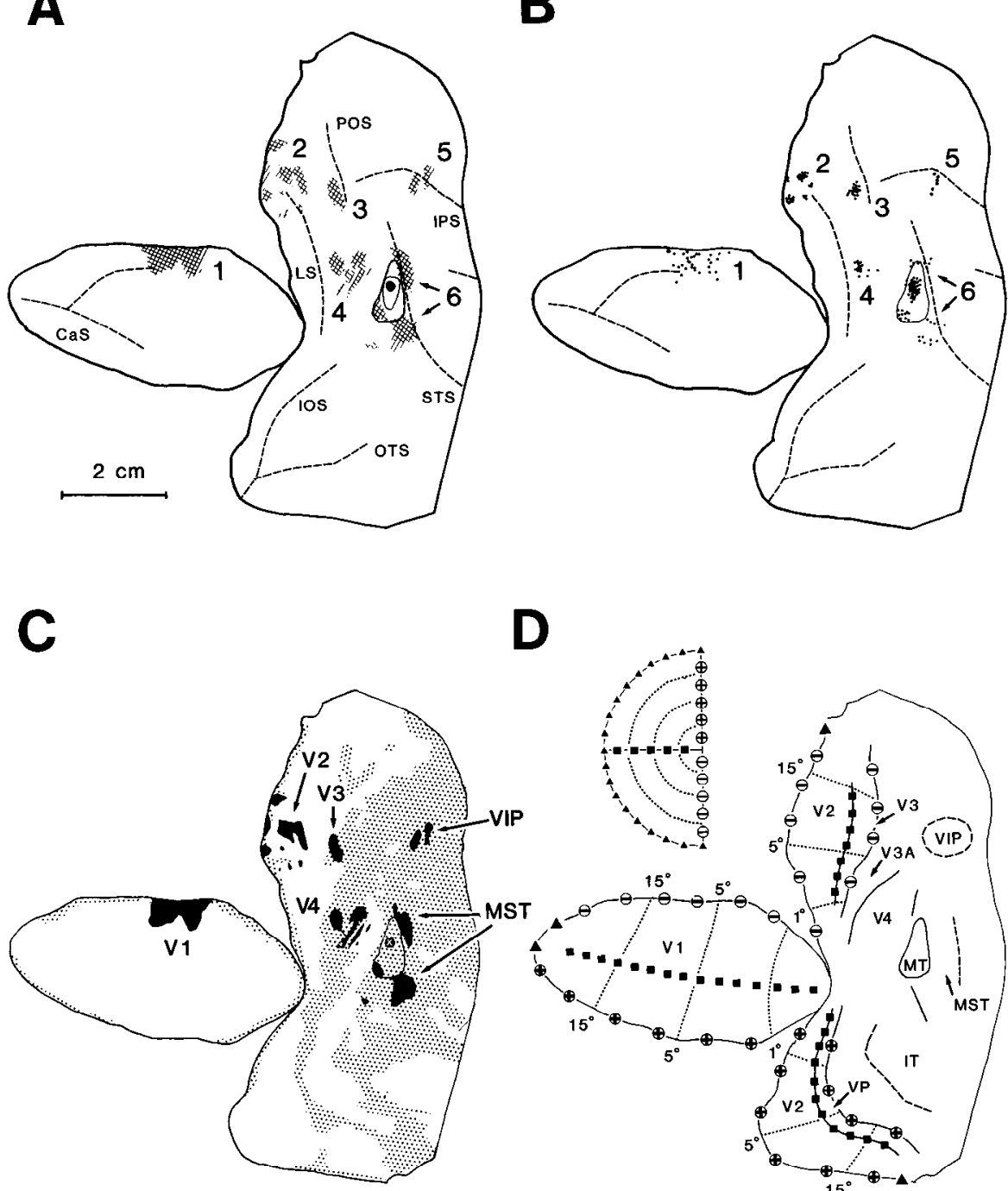

D

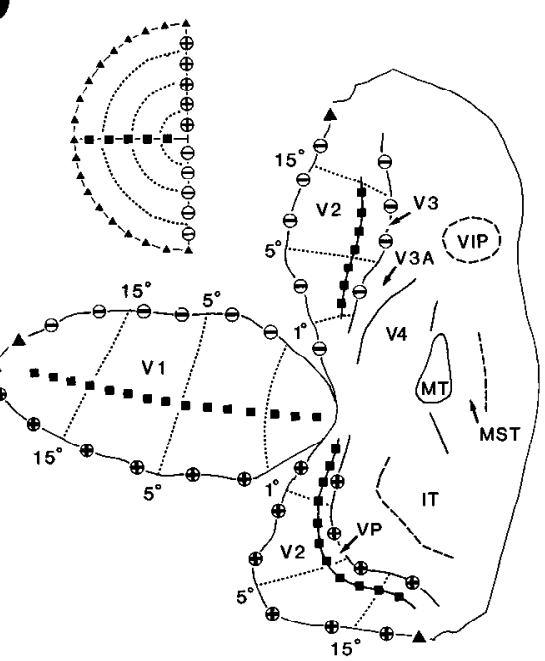

Figure 3. A, A two-dimensional map showing the sites of anterogradely transported label in visual cortex resulting from injection 1 . The location and extent of anterogradely transported label is indicated by hatching on a two-dimensional, unfolded map of visual cortex. The light solid line around the injection site indicates the region in which spread from the injection site obscured whatever transported label might have heen present. For convenience, the different labeled regions have been numbered. The fundus of each sulcus is marked with a dashed line and labeled using the following abbreviations: $C a S$, calcarine sulcus; IOS, inferior occipital sulcus; $I P S$, intraparietal sulcus; $L S$, lunate sulcus; OTS, occipitotemporal sulcus; $P O S$, parieto-occipital sulcus; STS, superior temporal sulcus. $B$, The pattern of retrogradely transported label in visual cortex from the same injection. The location of HRP-filled cells is shown by dots on a map of visual cortex. The numbering scheme for cortical connections is the same as that in $A$. The position of retrogradely transported label largely coincides with that of anterograde label, indicating that most cortical pathways to MT are reciprocal. $C$, The pattern of callosal inputs. Stippling indicates the location of degenerating axon terminals following transection of the splenium of the corpus callosum. For clarity, the fundi of the sulci have not been marked. The pattern of callosal inputs can be used to identify the position of several extrastriate visual areas. The locations of transported label are marked in solid black on this map, and most can be unambiguously assigned to specific visual areas on the basis of the callosal inputs. $D$, The location of established visual areas based on the pattern of callosal inputs. The locations of 10 visual areas in this hemisphere are indicated. These assignments are based on the pattern of callosal inputs in $C$. The approximate positions of horizontal and vertical meridian representations, and selected eccentricities, have been indicated for those areas with known topographic representations. 

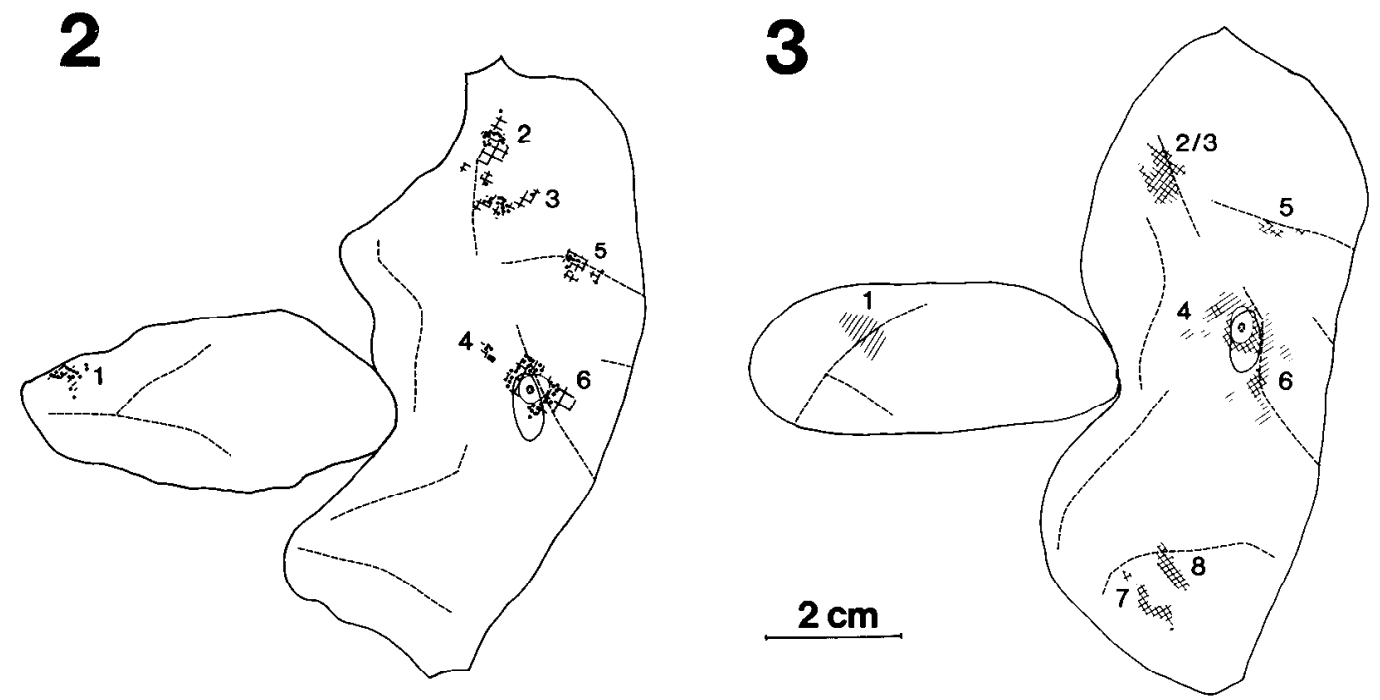

Figure 4. Two-dimensional maps of transported label for injections 2 and 3. Transported anterograde (hatched areas) and retrograde (dots) label are shown on two-dimensional maps of the visual cortex for injections 2 and 3 . The labeled regions have been numbered with the same conventions used in Figure 3. Open spaces around the injection sites indicate a "halo" of diffusion within which transport could not be scored. HRP data are not available for injection 3 . This injection, unlike the other two, resulted in two patches of label in ventral cortex (zones 7 and 8 ).

eration after transection of the splenium of the corpus callosum. The pattern for this hemisphere is similar to that seen previously (Van Essen et al., 1982) and is shown by stippling in Figure 3C. Regions labeled by the HRP or proline injections are marked in solid black. Figure $3 D$ is a map of the same hemisphere on which the borders of visual areas have been marked, based mainly on the relationship with the callosal connections. Figure $3 D$ also shows the approximate topographic representation expected in selected visual areas (Van Essen and Zeki, 1978; Newsome et al., 1980; Gattass et al., 1981; Van Essen et al., 1981, D. C. Van Essen, J. H. R. Maunsell, and W. T. Newsome, submitted for publication). There are 10 distinct areas, most of which have been shown to contain topographic representations of part or all of the contralateral visual hemifield. The foveal representation for most of these areas is near the center of the map (lateral cortex), with inferior fields dorsal and superior fields generally ventral (V3A and MT are exceptions). Several areas lack obvious topographic organization, including two, the medial superior temporal (MST) and ventral intraparietal (VIP) areas, which were not previously recognized and which have been identified in large part on the basis of results of the present study (see below).

The visual areas associated with each of the connections of MT are indicated by the labels in Figure $3 C$. In some regions the pattern of labeling is distinctly patchy, with distances of up to several millimeters between patches. The borders of some areas, such as V2 and V4, are sufficiently well defined to allow confidence that these are multiple patches within a single visual area rather than connections with distinct areas. Other patchy projections, such as those to MST and VIP, are considered unitary for reasons explained below.

Similar results were obtained from the other two in- jections. Figure 4 shows cortical maps for injection 2 and injection 3. As with injection 1, the distribution of label was extensive and complex, but it was possible to identify many connections corresponding to those found in the preceding example. Hence, labeled regions were numbered according to the same scheme. HRP data were not available for injection 3 , but for injection 2 the anterograde labeling (Fig. 4, hatching) and retrograde labeling (Fig. 4, stippling) were as clearly superimposed as in the case of injection 1 . Injection 3 , which was at the representation of the horizontal meridian, resulted in two patches of label ventrally, numbered 7 and 8 , which were not seen in the other cases. Figure 5 shows the pattern of callosal inputs (stippling) for all three hemispheres along with the labeled regions associated with each injection (black) and, whenever possible, the assignment of these regions to particular visual areas.

Given the extensive connectivity between MT and other regions of cortex, there is great value in identifying patterns that clarify the relationships within the overall network. One of the striking features of our results was that there are systematic differences in the laminar organization of connections between MT and other areas. The differences are like those which have been reported for many other reciprocal pathways-namely, in one direction a projection originates primarily from superficial layers and terminates mainly in layer IV, whereas the projection in the other direction originates in substantial part from infragranular layers and terminates mainly outside layer IV. In all instances in which a clear polarity can be inferred on other grounds, the first pattern is associated with forward projections (leading away from primary sensory cortex), and the second is associated with feedback (Tigges et al., 1977, 1981; Jones and Wise, 1977; Kaas et al., 1977; Rockland and Pandya, 1979). This correlation fits well with the present 


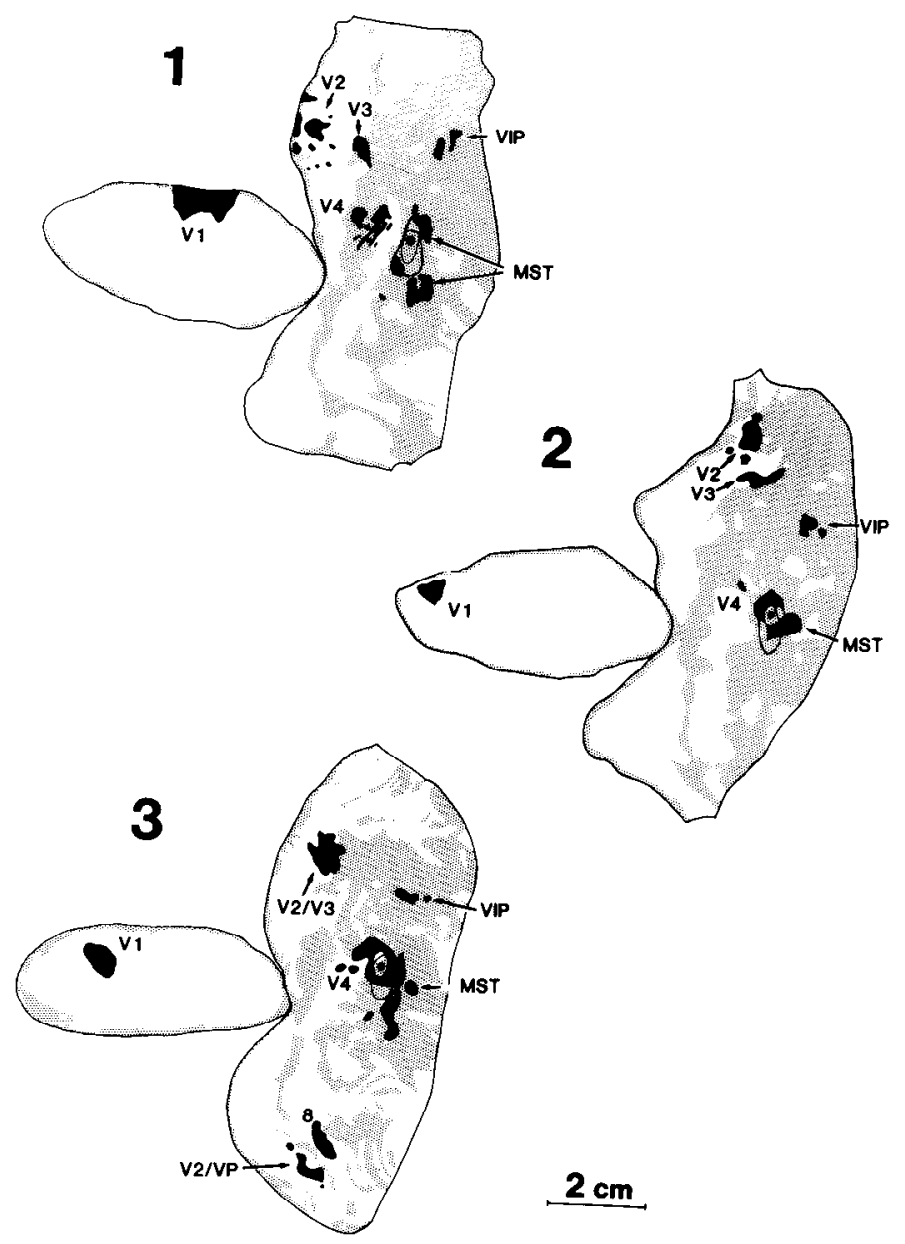

Figure 5. The pattern of callosal inputs on two-dimensional maps for all three injections. Stippling marks the location of callosal input in each map. The three patterns are similar. 'Transported label is marked in solid black. By using the pattern of callosal inputs, most of the label can be assigned to specific visual areas. The labeled zone which has not been assigned to specific visual areas is numbered as in previous figures.

results for MT, and it suggests an anatomical basis for assigning visual areas to well defined positions in an overall cortical hierarchy, as will be considered fully under "Discussion." We will use the terms forward and feedback in a provisional sense below, since they provide a convenient framework for a detailed description of connectivity patterns.

Table I shows the cortical layers giving rise to the projections to MT and those layers in which projections from MT terminate. For each area, the layers are listed in descending order of involvement. The laminar distributions of label within each area were consistent among the three injections. Figure 6 illustrates the distribution of anterogradely transported label in different visual areas after $\left[{ }^{3} \mathrm{H}\right]$ proline injections of MT. The complete pattern was not always obvious on individual sections, as the cortex was often sectioned obliquely; accordingly, a series of sections through cach labcled region was examined to determine all of the layers involved. Based on the criteria described above, it is possible to classify the areas connected with MT into a group of five which send forward projections to MT, a pair of areas which receive forward projections from $\mathrm{MT}$, and a single area, V4, which does not fit cleanly into either class, and which we have placed in an intermediate position (see below). We will consider each group of areas separately. Most of the outputs of MT that we identified have also been reported in an anterograde tracer study by Ungerleider et al. (1982). There are a few differences in results or interpretation, though, which will be mentioned in connection with the individual pathways as they are presented below.

Areas with forward projections to $M T: V 1, V 2, V 3, V P$ and connection 8 . It is known from previous studies that $\mathrm{V} 1$ and $\mathrm{MT}$ in the macaque are reciprocally connected and that the projection from V1 to MT arises from layer $\mathrm{IVb}$ and, to a much lesser extent, layer VI (Zeki, 1969; Lund et al., 1976; Weller and Kaas, 1978; Wong-Riley, 1979). 'The present experiments confirm these observations and, in addition, show that the projection from MT back to V1 terminates in precisely those layers from which the projection to MT originates, namely layers IVb and VI (Fig. 6, Table I). More than 90\% (52 of 56) of the retrogradely labled cells in V1 were in layer IVb; the remaining four labeled cells were large cells in the upper part of layer VI. The reciprocal pathway from MT to V1 was weak, and it was necessary to expose the autoradiography sections for 6 weeks to see the projection to layer VI at all. No projection was seen to layer I in V1.

It has previously been shown that V2 projects to MT (Zeki, 1971). It was also suggested in the same report that V3 projects to MT, but the existence of this pathway was less convincing due to uncertainties in determining the location of V3. We have found that both areas indeed do have a major projection to MT. The identification of the V3 projection in our matcrial appears unambiguous because of its precise relationship to the pattern of callosal inputs, with V3 situated immediately posterior and medial to the major callosal-recipient strip that runs

TABLE I

Laminar distributions of MT connections

\begin{tabular}{|c|c|c|}
\hline & $\begin{array}{c}\text { Laminar Origins of } \\
\text { Projections to MT }\end{array}$ & $\begin{array}{c}\text { Laminar Terminations of } \\
\text { Projections from MT }\end{array}$ \\
\hline V1 & IVb, VI & IVb, VI \\
V2 & III, II & I, VI, V, II, III \\
V3 & III, II & I, VI, V, II, III \\
VP & & VI, I, V, II \\
Conn. 8 & VI, V, I, II \\
\hline V4 & III, II, V & I, VI, II, III, V \\
\hline MST & II, II, III, IV, V, VI \\
VIP & VI, III, V & IV, III, II, V, VI \\
& & IV, III, II \\
\hline
\end{tabular}


between the lunate and the parieto-occipital sulci (Van Essen and Zeki, 1978). The projections arise mainly from layer III, but a few were also seen in the lower half of layer II (Table I). No labeled cells were found in the infragranular layers of either area ( 0 of 159 in V2, 0 of 89 in V3), indicating that the projection was forwardgoing to MT. The number of retrogradely labeled cells was largest in V2, with only a third to half as many in V1 and V3. This suggests that all three areas play a major role in providing visual inputs to MT, with V2 probably the most important. The reciprocal pathways, from MT to V2 and V3, were similar for both areas, with the laminar distribution of anterograde label indicative of a feedback projection. The density of label was greatest in layers I and VI, with less in layers II, V, and III (Fig. 6 ). Virtually no label was seen in layer IV. The laminar distribution of anterogradely transported label in V2 and V3 was the same for all three injections, even though injection 3 involved principally the deep layers of MT (Fig. 1). The distribution of anterograde label in both of these areas was patchy, as will be discussed further in another section.

Although all three injections revealed connections with V2 and V3 dorsally, only injection 3 produced label in ventral extrastriate cortex. One focus of label was at the border between VP and ventral V2, each of which con-
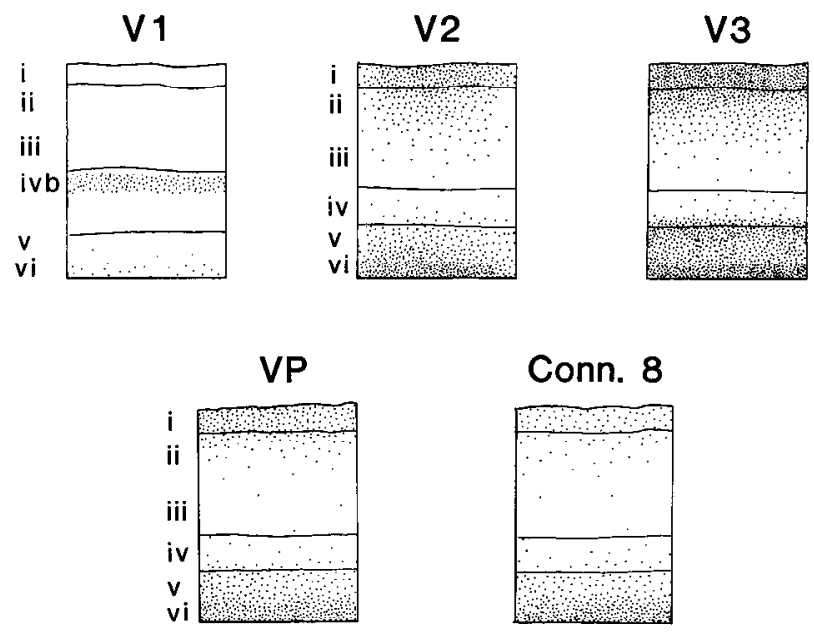

Conn. 8

\section{FEEDBACK}
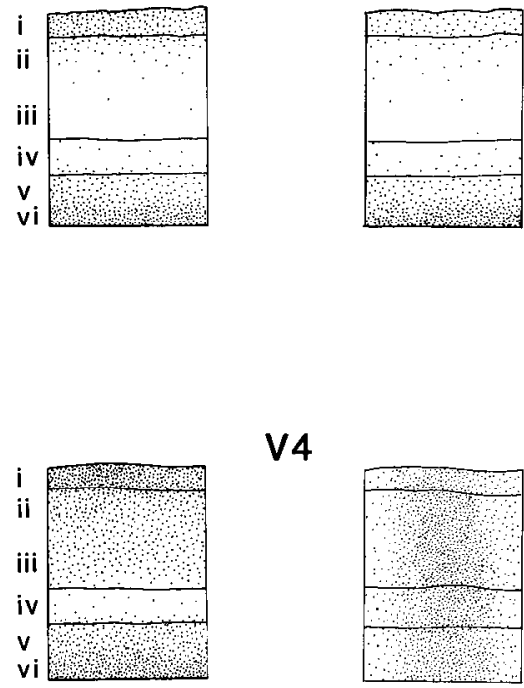

V4

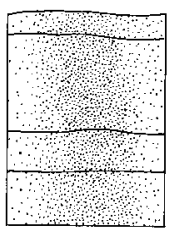

\section{INTERMEDIATE}
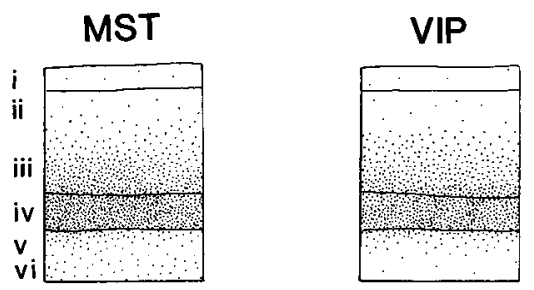

\section{FORWARD}

Figure 6. The laminar distribution of anterogradely transported label. The laminar distribution of label is shown for each of cortical targets of MT. Feedback projections from MT are characterized by terminating largely in layers I and VI and sparing layer IV. Forward projections terminate mainly in layers IV and III. Two different laminar distributions were found in V4, one sparing layer IV and the other labeling all layers approximately equally (see the text). 
tains a representation of the superior visual quadrant. As mentioned above, this additional label is attributable to the injection site being centered on the horizontal meridian and extending into both the superior and inferior field representations. At its closest, the label was only about $1 \mathrm{~mm}$ from the center of the callosal-recipient strip which marks the anterior border of VP (Newsome et al., 1980). At its farthest, the label was about $5 \mathrm{~mm}$ from the center of the callosal-recipient strip. This projection is likely to include both V2 and VP, since VP is several millimeters wide based on physiological and anatomical estimates of the separation between horizontal and vertical meridian representations (Newsome et al., 1980). However, there may be substantial variability in the width of VP; thus, this interpretation is not conclusive. The autoradiographic label at the V2/VP border was found principally in layers VI and I, with some spread into layer V. Since the HRP injection was not successful, we could not ascertain whether the connection with MT was reciprocal in this animal, but recent experiments from this laboratory indicate that $\mathrm{VP}$ indeed has a forward projection to MT (A. Burkhalter and D. C. Van Essen, unpublished results).

Injection 3 also labeled cortex in a second region of ventral extrastriate cortex (connection 8 , Fig. 5). This label was within a callosal-free zone which is a regular feature in the pattern of callosal degeneration. It is unclear whether this region is part of V4 or whether it consists of one or more distinct visual areas (Van Essen et al., 1982). Because of this uncertainty, we will refer to it simply as connection 8 of MT. The laminar distribution of radioactive label differed from other feedback projections from MT in that there was little involvement of the superficial layers; most of the label was in layer VI, with some spread into layer V. This distribution is different from that in V1 of the same hemisphere (see below), providing some support for the notion that connection 8 does involve cortex that is distinct from V4.

Forward projections from MT to areas MST and VIP. Two cortical areas were classified as receiving forward projections from MT and returning feedback projections to it. A major projection from MT is to a region immediately medial to it, along the fundus of the superior temporal sulcus and in places extending several millimeters onto the anterior bank. This region is known to contain a high percentage of direction-selective neurons with receptive fields much larger on average than those in M'I (Newsome and Wurtz, 1981; Van Essen et al., 1981). In addition, this region has been shown to project to area $7 \mathrm{a}$, or PG (Mesulam et al., 1977), and to the frontal eye fields (Barbas and Mesulam, 1981). In one of our injections the projections medial to MT terminated in discrete patches up to $6 \mathrm{~mm}$ apart, but in the other two cases the labeling was continuous, or nearly so. Patchiness of the labeling pattern has also been reported in the same region for some, but not all cases in the aforementioned studies on parietal and frontal lobe connections. Since distinct foci are not a consistent feature of either the input or the outputs of the region medial to MT, there does not appear to be sufficient justification for subdividing it into multiple areas. We therefore consider MT-recipient cortex medial to MT to be a single area, which we have designated the medial superior temporal visual area (MST). Autoradiographic label in MST was found primarily in layer IV with less in layers II and III, and still less in layers V and VI, indicative of a forward projection. HRP-labeled cells were mainly in deep layers (69 of 100), indicative of feedback.

Another target of MT was a region of cortex in and near the fundus of the intraparietal sulcus. There is also a projection to what appears to be the same region from ventral V2 (our unpublished observations). On the basis of these connections, as well as its pattern of callosal inputs, this region can be distinguished from areas V3 and V3A, which lie posterior to it (Van Essen and Zeki, 1978), from POa, which lies dorsal to it in the lateral bank of the intraparietal sulcus (Seltzer and Pandya, 1980 ), and from PEa, an architectonic subdivision on the medial bank of the intraparietal sulcus (Pandya and Seltzer, 1982). Although little else is known about this region, these features appear sufficiently distinctive to regard it as a separate cortical area, and we have designated it the ventral intraparietal area (VIP). The laminar distribution of label in VIP was of the forward pattern, similar to that in MST, the only difference being that VIP had virtually no anterograde label in layers $\mathrm{V}$ and VI (Fig. 6). Only a few retrogradely labeled cells were found in VIP, but most of them (5 of 8 ) were in deep layers, suggesting a feedback pattern.

Ungerleider et al. (1982) reported two distinct projections of MT to the cortex in the intraparietal sulcus. The more anterior of their projections corresponds well with the projection we found to VIP. However, we did not find an additional projection zone posterior to VIP in the intraparietal cortex, except in the case of injection 2, where the projection to V3 was at the juncture of the intraparietal and parieto-occipital sulci. The reason for this apparent discrepancy is unclear.

The connection of MT with V4. Each of the three MT injections led to transported label in the region designated as connection 4 in Figures 3 and 4 . For injection 1 , the label was concentrated on the anterior bank of the lunate sulcus, directly posterior to M'T and extending from near the fundus of the sulcus out onto the prelunate gyrus. Most of the labeled patches were within the broad strip of callosal-recipient cortex which runs through this part of the lunate sulcus and which itself is contained mainly within V4 (Van Essen and Zeki, 1978; Zeki, 1978; Van Essen et al., 1982). For injections 2 and 3 , the connection 4 label was less intense and was located on the pre-lunate gyrus and in the superior temporal sulcus dorsal and lateral to MT. Once again, the relationship to the pattern of callosal inputs suggested that most or all of the label was within V4. The assignment for these two injections should be regarded as provisional, however, because there are major uncertainties concerning the full extent of V4 (see Van Essen et al., 1982).

The pathway between MT and V4 was the only one which did not fit cleanly into the pattern of a forward laminar distribution in one direction and a feedback distribution in the other. in one direction, from V4 to MT, the pattern seemed straightforward and indicative of a forward projection from $\bigvee 4$ to $\mathrm{MT}$. The labeled cells in V4 following both of the successful HRP injections 
were nearly all supragranular (53 of 55), mainly in layer III, but with a few in the lower half of layer II. However, in the reverse direction, two different laminar distribution patterns were seen in the anterograde transport to $\mathrm{V} 4$, as indicated in Table $\mathrm{I}$ and Figure 6. For each injection, some of the patches of anterograde label in V4 had a feedback distribution, with most label in the superficial and deep layers, whereas other patches were in the form of radial columns, with relatively uniform labeling of all layers. Following injection 1 the most heavily labeled patch, near the fundus in the anterior bank of the lunate sulcus, had a feedback distribution, but the other patches further dorsal in the anterior bank were columnar in appearance. Similarly, for injection 3 , the most dorsal patch in V4, also the most heavily labeled, had a feedback distribution, while the remaining patches were columnar.

The regional differences in laminar distribution are consistent with other lines of evidence for heterogeneity within V4 (Zcki, 1971, 1977; Van Essen and Zeki, 1978; Schein et al., 1982). Resolving whether the different laminar patterns occur in functionally distinct subdivisions of $\mathrm{V} 4$ will obviously require more extensive investigation. In the meantime, we will tentatively consider the relationship between $\mathrm{M}^{\prime} \mathrm{I}$ and $\mathrm{V} 4$ as intermediate in type (see "Discussion").

Topographic relationships and patchiness of intrinsic, extrinsic, and contralateral connections. The connections of MT vary not only in the laminar distribution of label, but also in size (both in absolute extent and relative to the overall size of an area) and in the degree of patchiness resulting from focal injections. This brings up several interesting issues in relation to topographic order among the connections. One would expect a priori that any given site within MT would have its strongest connections with topographically corresponding portions of other visual areas. Since we knew the location and size of receptive fields for the injection sites in MT, it was possible to make useful comparisons with the location and extent of the connections with other areas. The labeled pathways to and from all visual areas known to be topographically organized (V1, V2, V3, and VP) were found to connect portions of these areas containing the representation of the same part of the visual field as that at the injection. The orderliness of these connections can be seen in Figure 5. In V1, increasing eccentricity at the injection site (injection $1<$ injection $3<$ injection 2 ) was coupled with label progressively further from the foveal representation (at the right), each case corresponding well with the expected representation of its eccentricity in V1 (Daniel and Whitteridge, 1961; D. C. Van Essen, J. H. R. Maunsell, and W. T. Newsome, submitted for publication). Also, injections at sites further from the vertical meridian resulted in label further from the dorsal border of V1, where the vertical meridian is represented. It is worth noting that neither of these relationships would have been clearly discernible if we had been forced to rely on a standardized map of visual topography in MT, rather than on recording data from each animal.

Similar orderliness existed in the connections with V2, $\mathrm{V} 3$, and VP. Injection 3 resulted in label further from the foveal representation than injection 1 , and that from injection 2 was further still. Injection 1 was near the representation of the vertical meridian and resulted in label at the posterior (left) border of $\mathrm{V} 2$ and the anterior (right) border of V3, where the vertical meridian is found in each area. For injection 2, further from the vertical meridian, the label in V2 and V3 was closer to their common border at the representation of the horizontal meridian. Finally, injection 3, which included the representation of the horizontal meridian and superior fields, resulted in label in the expected locations at the V2/V3 border dorsally and the V2/VP border ventrally.

It is naturally of interest to know whether this set of injections provides evidence for topographic order in other, less well characterized visual areas. One area whose connections did suggest some topographic order was V4. The label from injection 1 , closest to the vertical meridian, was almost entirely within the callosal-recipient portion of $\mathrm{V} 4$, whereas that from injection 3, along the horizontal meridian representation, extended well into the callosal-free portion of presumed V4. The injection closest to the foveal representation (injection 1) produced label mainly within the lunate sulcus portion of V4, whereas the more peripheral injections (2 and 3) produced label closer to MT, on the pre-lunate gyrus and in the dorsal-lateral part of the superior temporal sulcus. Together, these injections suggest a crude topographic organization in which the inferior vertical meridian is represented in callosal-recipient cortex along the dorsal margin of V4, with the fovea posteroventral (down and to the left on the map) and the periphery anterodorsal (up and to the right on the map). It is not clear from our results or from previous studies whether superior fields are represented anywhere within V4.

Only injection 3 labeled ventral cortex in the cullosalfree zone anterior to VP (connection 8). This observation, coupled with the relationship of the labeled region relative to callosal-recipient cortex, leads to a plausible suggestion for the topographic organization of cortex in the vicinity of connection 8 . The absence of projections from the other two MT injections indicates that the inferior quadrant is not represented in this region. Injection 3 demonstrates that the horizontal meridian is represented approximately midway across the callosal-free zone, and it is likely that the superior vertical meridian is represented along the callosal-recipient strips anterior as well as posterior to this zone. These observations suggest that there is a dual representation of the superior quadrant in this region, with a topographic organization analogous to that already described for VP and ventral $\mathrm{V} 2$, the two areas occupying the callosal-free region posterior to connection 8 (Newsome et al., 1980).

The projections to MST and VIP were to somewhat different locations in each case, but there is no obvious topographic organization suggested by the results. Given the very large size of receptive fields in MST (Van Essen et al., 1981), it is possible that topographic organization has been largely or entirely eroded by this stage of processing. Alternatively, these areas may have a crude topographic organization that is obscured by a high degree of individual variability or by connections which are not restricted to corresponding portions of the visual representation. 
The extent of label differed in the various visual areas in a manner consistent with the different magnification factors of their visual field representations. Although the injections in MT were reasonably focal $(<2 \mathrm{~mm}$ diameter), retrograde and anterograde label in V1 occurred over a fairly large region, 22 to $70 \mathrm{~mm}^{2}$ in surface area. The labeled region was roughly circular in one case and somewhat elongated in the other two. Based on the known magnification factors in V1 (Daniel and Whitteridge 1961), the extent of label in V1 corresponds to $27^{\circ 2}$, $430^{\circ 2}$, and $50^{\circ 2}$ for injections 1,2 , and 3 , respectively. This can be compared to the receptive field sizes recorded at the injection sites in MT of $20^{\circ}, 340^{\circ}$, and $60^{\circ 2}$ (Fig. $2 B$ ). The agreement is quite good, given that there is significant individual variability in magnification factors

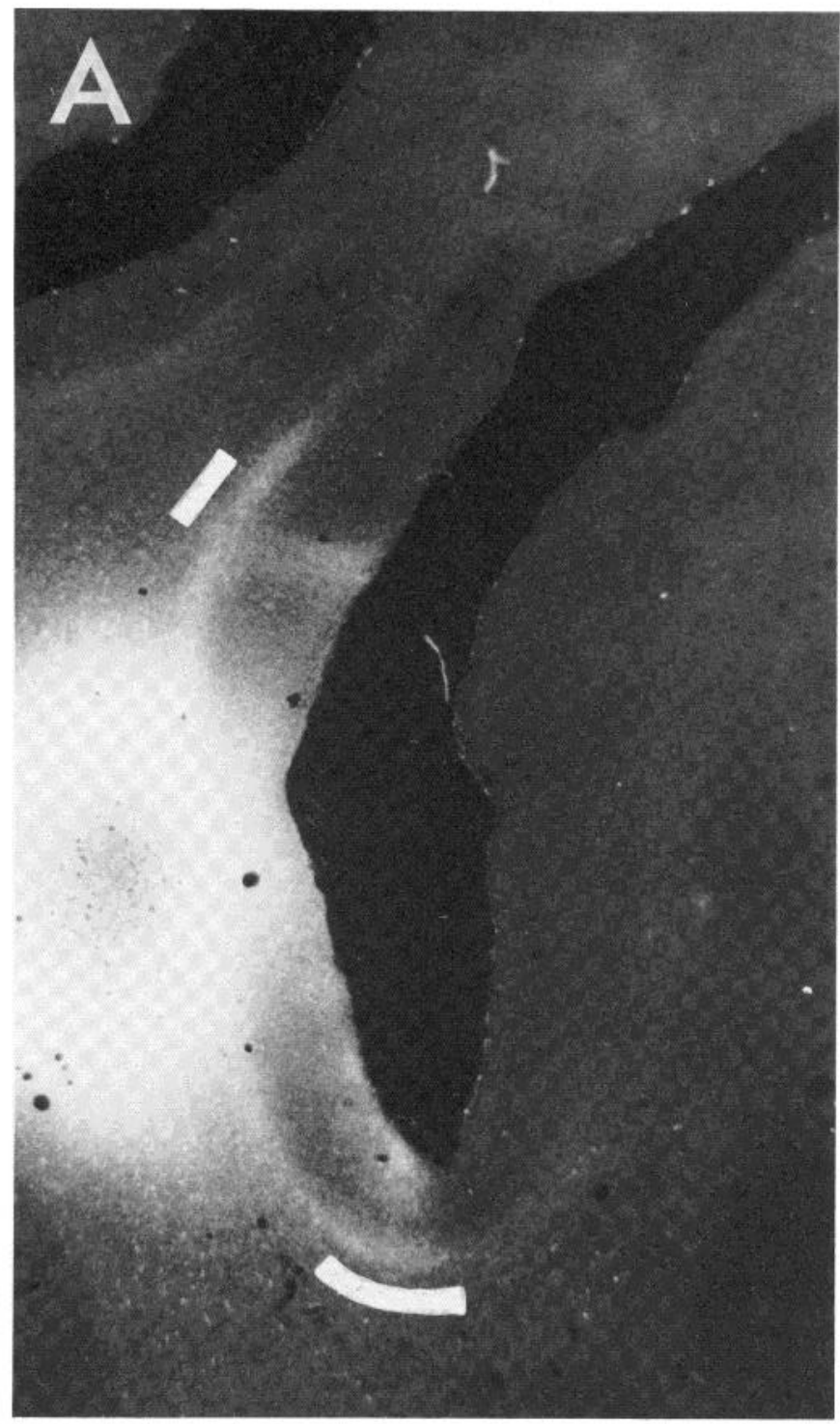

in V1 (D. C. Van Essen, J. H. R. Maunsell, and W. T. Newsome, submitted for publication). Thus, the direct inputs from V1 are extensive enough to account for the main excitatory drive to MT neurons.

The tracer injections also revealed extensive connections within MT. These intrinsic connections were widespread enough to involve a much larger portion of the visual field than that occupied by receptive fields at the injection site. The distribution of label was different in each case, perhaps as a result of differences in the particular layers injected. Injection 1 resulted in a conspicuous patch of retrograde and anterograde label near the ventral tip of MT, separated from the injection site by a region with very little label (Fig. 3 ). The heavily labeled patch was clearly within the architectonic border of MT.

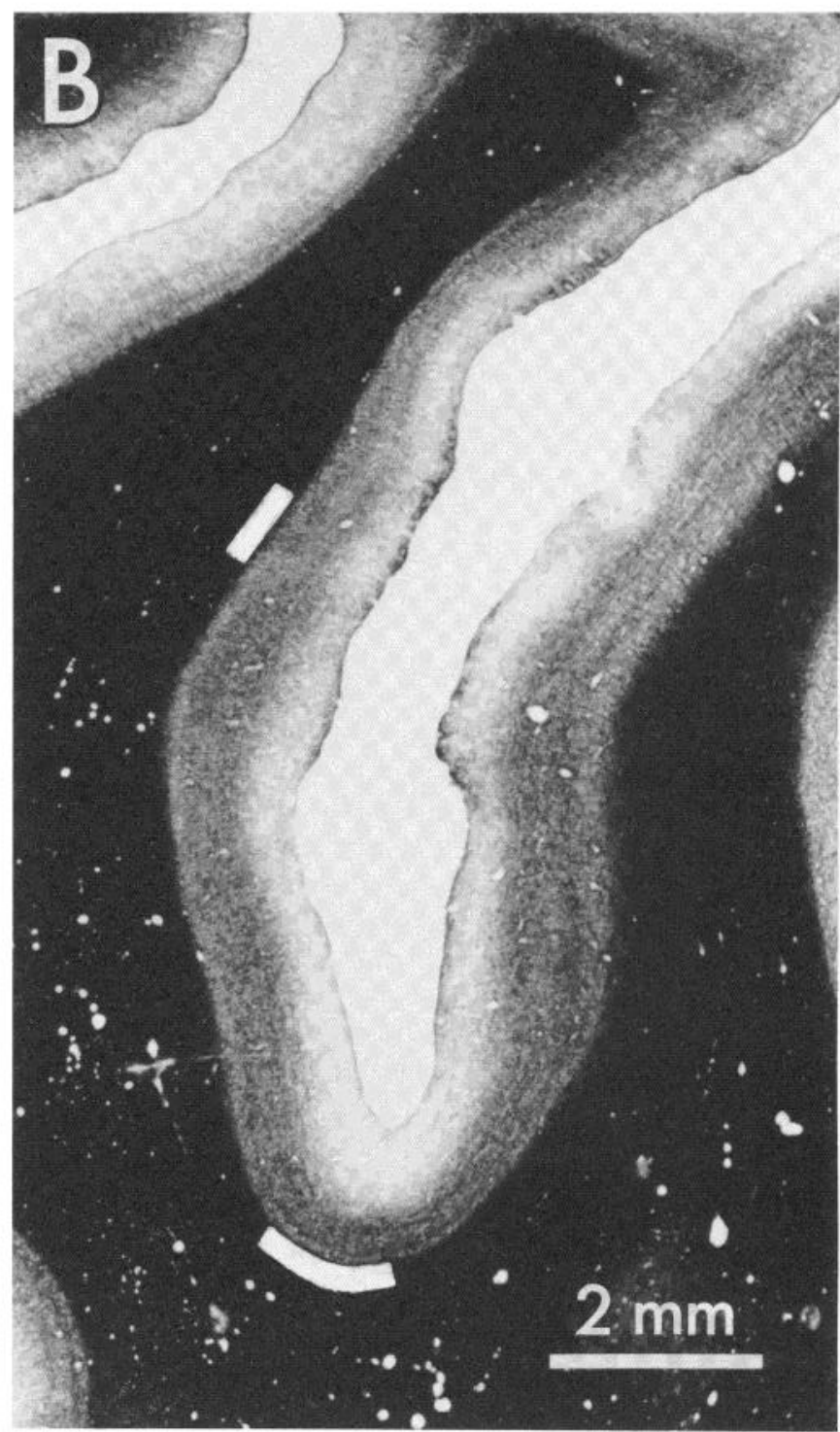

Figure 7. Intrinsic connections of MT following injection 3. A, A darkfield photomicrograph of a horizontal section, taken 1 $\mathrm{mm}$ ventral to the center of the injection, which was processed for autoradiography. There is heavy labeling of layer VI and lighter labeling of superficial layers extendng a long distance from the injection. $B$, A nearby section which was stained for myelin and which was used to find the borders of MT. White bars below the cortex indicate the range of uncertainty in the position of each border on this section. The transition in myelination is visible for the lateral (upper) border, but on this section the medial border is not obvious at this magnification. White bars have been drawn on the section $A$ to indicate the corresponding positions. The label in layer VI extends beyond the lateral border of MT. On other sections both the superficial and deep label crossed this border. 
The anterograde label was distributed through all cortical layers whereas the retrogradely labeled cells were mainly in layer III ( 20 of 23$)$, with a few cells ( 3 of 23 ) in layer V. A similar intrinsic connection was seen in one case by Wall et al. (1982) following an injection of MT in the bushbaby.

Neither of the other two injections resulted in a discrete patch of label within MT. Injection 2 was surrounded by retrograde and anterograde label whose density decreased steadily with distance, and which was not obviously concentrated in any layer, making it difficult to distinguish the relative contributions of diffusion versus local transport. However, injection 3 had a different and striking pattern of intrinsic connections. This injection was centered in the white matter directly below MT and involved mainly the deep layers. The distribution of anterograde label is shown in the darkfield photomicrograph of Figure $7 A$. Figure $7 B$ is a photomicrograph at the same magnification of a nearby section which was stained for myelin. The borders of MT can be seen in this section, and the range of uncertainty in their positions is indicated by white lines below the cortex. I ayer VI is brightly labeled throughout the width of MT, and the label clearly extends beyond the lateral border. The labeled strip terminates in discrete columns extending continuously into the superficial layers. This pattern of labeling was seen for a dorsoventral extent of $4 \mathrm{~mm}$ around the injection site. One explanation for the particularly extensive labeling within layer VI in this case is that the injection involved mainly the infragranular layers. Widespread intralaminar labeling has also been seen following injections in somatosensory cortex (Jones et al., 1978).

The corpus callosum was not cut until several days after the injections were made, allowing time for transport of label to the contralateral hemisphere. Only injection 1 resulted in label in the contralateral hemisphere. Faint radioactive label was seen in the opposite MT and MST, but no HRP-labeled cells were found. A reciprocal projection presumably exists but was not detected due to the consistently lower degree of retrograde labeling in these experiments. The laminar distribution of label in the contralateral MST was the same as that in the ipsilateral MST, namely, densest in layers IV and III. The contralateral MT had a single patch of label extending the full thickness of the cortex, with no obvious concentration in any particular layer.

The labeling in the superior temporal sulcus of the contralateral (left) hemisphere following injection 1 is illustrated on a cortical map in Figure $8 A$. This map has been transposed so that it has the same configuration as the map of the injection site in the right hemisphere, shown in Figure $8 B$, i.e., with the fundus of the sulcus to the right of MT in both cases. The label in the contralateral MT was near its center, in a position roughly similar to that of the injection site in the other hemisphere, and the label in the contralateral MST was in a position corresponding to one of the labeled patches in the ipsilateral MST.

The occurrence of a projection from near the middle of MT in one hemisphere to the middle of MT in the opposite hemisphere raises the possibility that the pathway interconnects mirror-image portions of the visual
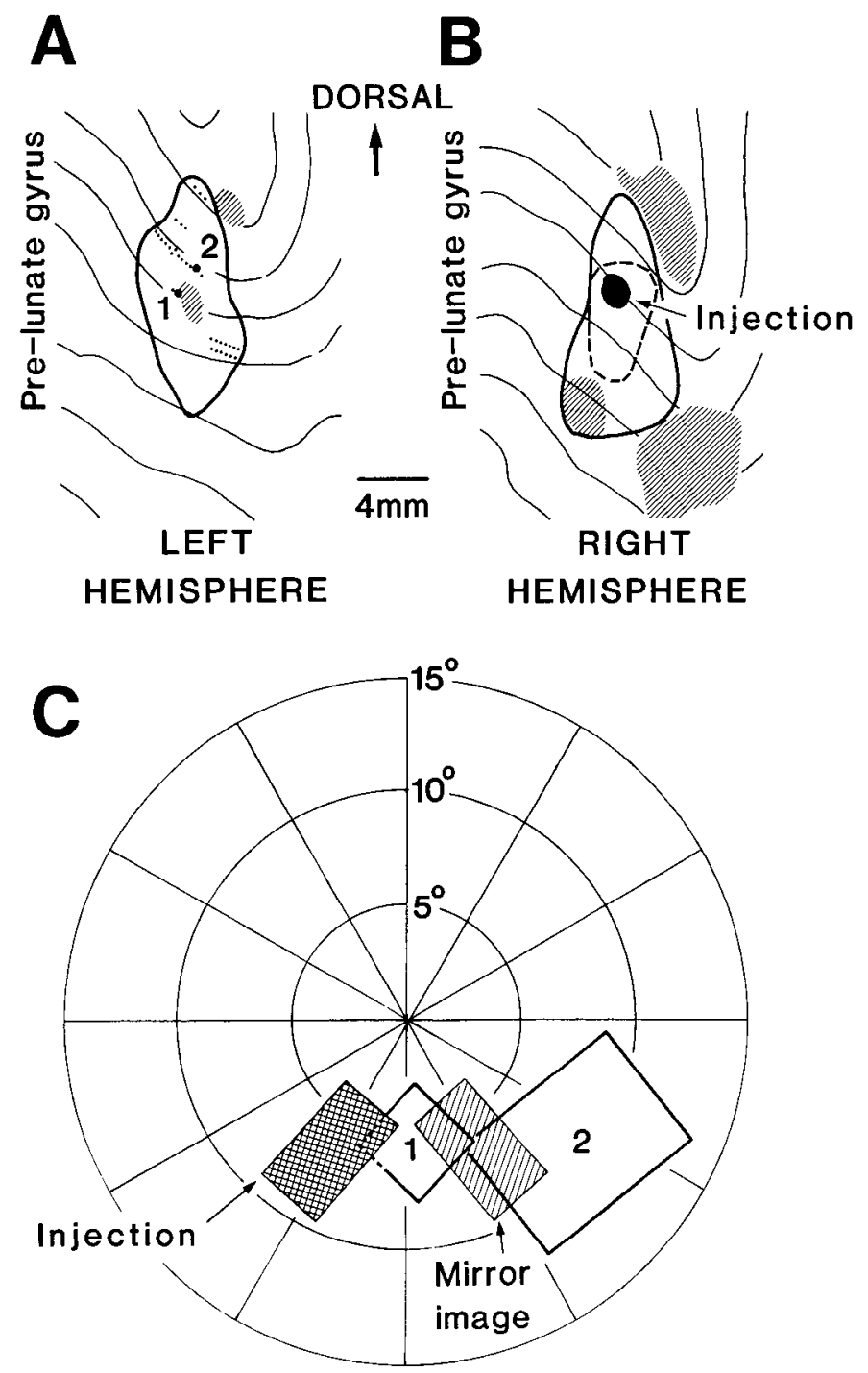

Figure 8. Contralateral transport of label following injection 1. A, A two-dimensional map of MT in the hemisphere contralateral to the injection. The map has been transposed so that it has the same orientation as maps of the other hemisphere. The border of MT is marked with a solid line, and the location of transported label is shown with hatching. Dots indicate sites at which receptive fields were plotted in a physiological recording session. $B$, The location of the injection site and nearby transport of label for injection 1 . The dashed line indicates the extent of spread of label from the injection site. The injection site and one of the patches of label in MST (to the right of the top of MT) occupy positions corresponding to the location of label in the contralateral hemisphere $(A) . C$, The locations of receptive fields for cells near the injection site and in the contralateral MT. The receptive field plotted at the time of the injection is crosshatched in the left visual hemificld. Its mirror image across the vertical meridian is hatched in the right hemifield. The receptive fields recorded at points 1 and 2 in the MT contralateral to the injection are also drawn. Since recording site 1 , but not 2 , was associated with transported label, the contralateral pathway is likely to link representations of similar parts of the visual field near the vertical meridian. Because receptive fields were plotted as rectangles, the left corner of the field plotted at site 1 may be drawn slightly further into the left hemifield than the actual response extended. On the other hand, the corpus callosum was cut before fields 1 and 2 were plotted, and it is possible that the receptive field at site 1 originally extended even further into the left hemifield. 
field. However, it is difficult to draw conclusions from these anatomical results alone, because of the known disorderliness in the topographic organization of MT. The receptive field at the injection site had been determined at the time of the injection, and a separate physiology session was carried out in this animal with the aim of mapping visual topography in the contralateral hemisphere. Receptive field plots were obtained at 33 recording sites, indicated by dots in Figure $8 A$. The receptive field recorded at the time of injection is drawn cross-hatched in the left hemifield in Figure $8 C$; its mirror image across the vertical meridian is hatched in the right hemifield. Two of the recording sites in the uninjected hemisphere were right at the margin of the patch of transported label. Their receptive fields, as shown in Figure $8 C$ for the site labeled 1 , were near the inferior vertical meridian. Of all the recordings from this hemisphere, these fields were closest to the receptive field of the contralateral injection site. In contrast, many of the recording sites (15 of 33 ) had receptive fields which partially overlapped the mirror image of the injection receptive field, and many of these sites were far from the transported label. The recording site labeled 2 in Figure $8 \mathrm{~A}$ and its receptive field in Figure $8 C$ are an example of these. These results are consistent with the notion that the interhemispheric connections of MT preferentially link regions representing nearby parts of the visual field. It may also be significant that there was no detectable transport to the opposite hemisphere from the other two injections, which involved representations well away from the vertical meridian. This evidence against the mirror-image hypothesis is not decisive, however.

The label within individual cortical visual areas was

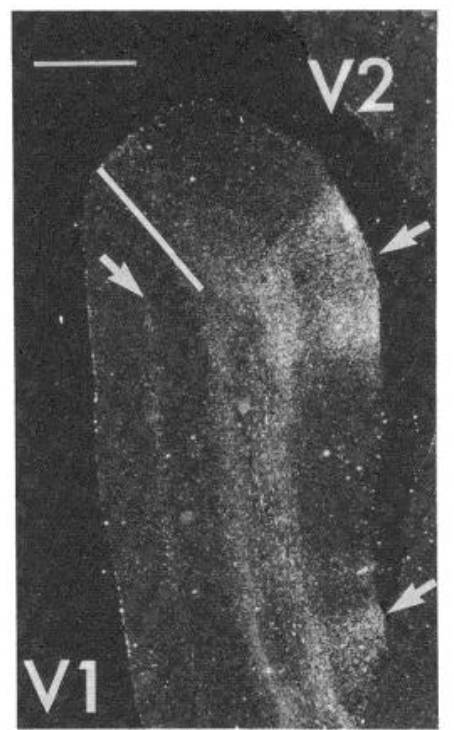

Figure 9. A darkfield photomicrograph of label in V1 and V2 following injection 1 in MT. A white line marks the border between V1 and V2. The arrow in V1 points to a faint uniform band of labeling in layer IVb of V1. Other arrows indicate two widely separated patches of label in V2. Label in V2 is mainly superficial and deep and does not extensively involve layer IV. The label in layer VI of V1 is too faint to be readily visible in this photograph. often distributed in patches, but the degree of patchiness varied from one area to the next. The two extremes were V1, in which the label was almost uniformly distributed, and V2, in which small, intensely labeled patches were separated by wide regions with little or no label. Figure 9 shows this contrast in a darkfield photomicrograph of anterograde transport in a section from the hemisphere of injection 1. The arrow on the left points to faint, evenly distributed label in layer IVb of V1. The arrows on the right indicate two widely separated patches of label in V2. A similar pattern of patchiness in V2, but not in V1, was found for the retrogradely labeled cells in the same experiment. This is indicated on two-dimensional maps of the relevant portions of V2 and V1 in Figure 10. The labeled cells in V2 were found in five distinct patches, which together covered roughly the same extent as that which was uniformly labeled in V1. For the other two injections, the patchiness in V2 was not as striking. Pronounced patches were also seen in V4, particularly in the case of injection 1 . Less dramatic patches were present in all of the remaining ipsilateral connections (Fig. 5). The functional significance of these patterns will be considered under "Discussion."

\section{Subcortical connections}

Descending projections were seen to eight ipsilateral subcortical centers. No contralateral subcortical projections were identified, and only one pathway, that to the pulvinar, had detectable reciprocal connections with MT. The apparent lack of inputs from various other subcortical structures which project to MT in other species (Spatz, 1975; Tigges et al., 1982) is almost certainly a reflection of the suboptimal conditions for HRP labeling in our experiments.

Claustrum and basal ganglia. Projections were seen to the claustrum, caudate nucleus, and putamen. The strongest of these was to the claustrum. Label was found in several small patches ( 0.5 to $1 \mathrm{~mm}$ diameter) which were distributed over about $5 \mathrm{~mm}$ along its posterior margin. The location of this label for injection 3 is diagrammed in Figure $11 B$.

Patches of similar size, but less intensely labeled, were found over about a 7-mm extent in the tail of the caudate nucleus. A section from the hemisphere of injection 3 which had label in the caudate is outlined in Figure $11 A$. Weak label was seen in the posterior part of the putamen (Fig. 11B). This label extended over about $1.5 \mathrm{~mm}$ and was not divided into obvious patches.

Thalamus. All injections demonstrated anterograde projections from MT to the pulvinar complex, the pregeniculate nucleus, and the thalamic reticular nucleus. By far, the densest thalamic label was in the pulvinar complex. We were particularly interested in ascertaining whether MT connections are restricted to a single, small subdivision of the pulvinar complex, as Lin and Kaas (1980) demonstrated for the owl monkey and Standage and Benevento (1983) recently reported for the macaque. The region described by Standage and Benevento (1983) is a narrow, crescent-shaped zone, approximately $5 \mathrm{~mm}$ long (dorsoventrally) and 1 to $2 \mathrm{~mm}$ wide (mediolaterally), which crosses the brachium of the superior colliculus and is restricted to the medial portions of the 


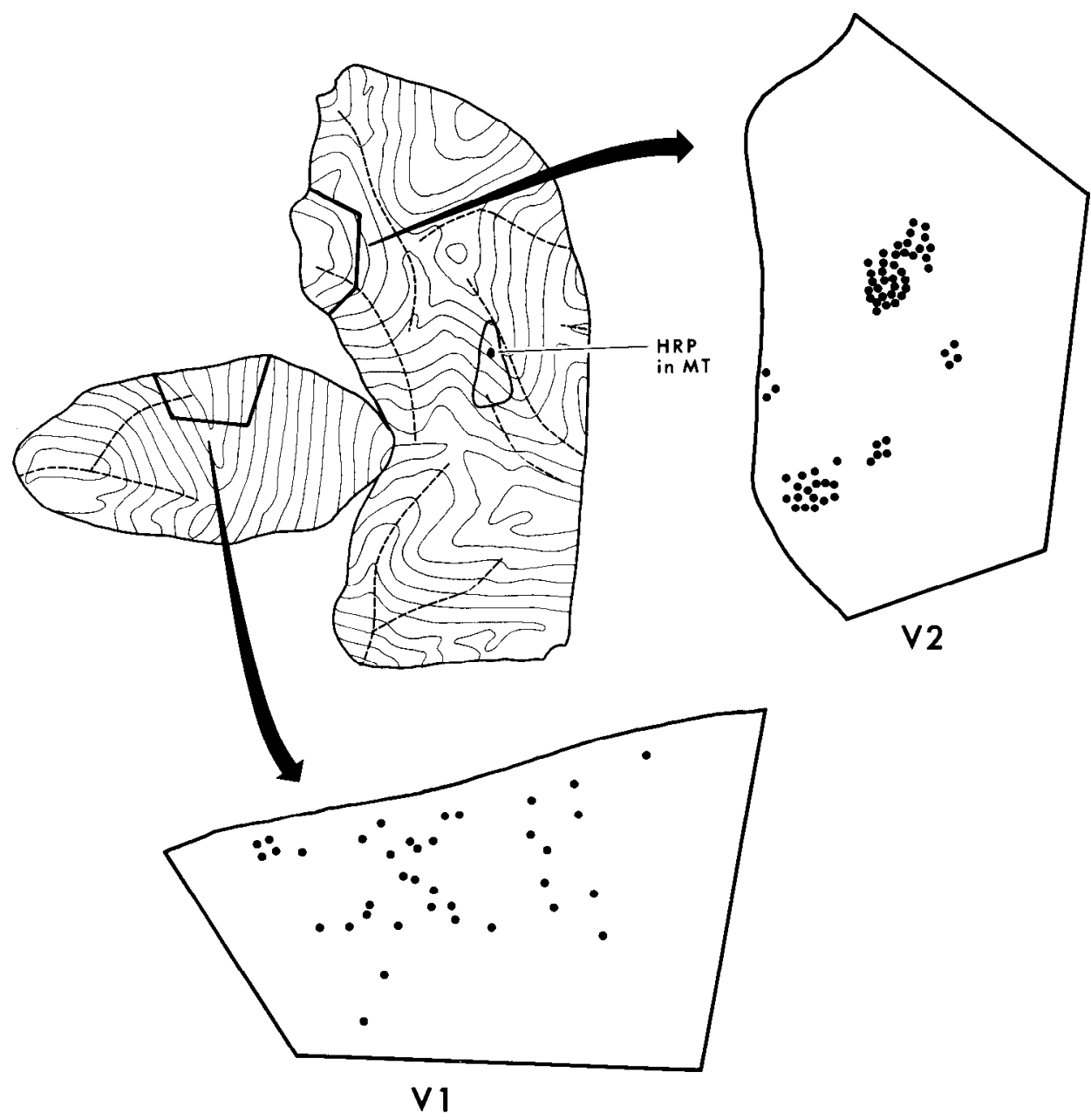

Figure 10. Two-dimensional maps of the distribution of HRP-labeled cells in V1 and V2 after injection 1. The label in V1 is evenly distributed over a large region. The label in V2 is found in five discrete patches, which collectively cover about the same extent as the label in V1.

inferior and lateral pulvinar. We found anterograde and retrograde label in roughly the same region of the pulvinar, but extending over a considerably greater mediolateral extent.

Figure 12 shows darkfield photomicrographs of autoradiographic sections from two of the experimental hemispheres. In Figure $12 A$ (injection 1, parasagittal section), two major patches of label are evident in a strip extending from the inferior pulvinar (PI), across the bracium of the superior colliculus $(B r S C)$, and into a portion of the lateral pulvinar (PL) just dorsal to the PI. Reconstruction of the entire labeled region from this hemisphere indicated that its overall dimensions were approximately $5 \mathrm{~mm}$ dorsoventrally, $3.5 \mathrm{~mm}$ mediolaterally, and $2.5 \mathrm{~mm}$ anteroposteriorly. In Figure $12 B$ (injection 3, horizontal section), most of the label is in two patches within the inferior pulvinar, at the juncture with the brachium of the superior colliculus. At this level, the lateral pulvinar contains only a faint patch of label (Fig. $12 B$, wide white arrow) posterior and lateral to the PI. Examination of sections dorsal to the one illustrated revealed more extensive label continuous with this patch in the PL, and also showing continuity with the label in the PI. Again, the full extent of the labeled zone was greater in both the mediolateral $(5 \mathrm{~mm})$ and the dorsoventral $(5 \mathrm{~mm})$ extent than along the anteroposterior axis $(4 \mathrm{~mm})$. In the remaining case (injection 2, not illustrated), there was once again patchy labeling within a region extending from the PI to the PL. The labeled region was slightly more medial than in the other cases and more elongated dorsoventrally $(5.5 \mathrm{~mm})$ than medio laterally $(2.5 \mathrm{~mm})$ or anteroposteriorly $(3 \mathrm{~mm})$.

Altogether, these results indicate that MT is connected with a large portion of the inferior pulvinar complex, which itself is a somewhat irregularly shaped region no more than $5 \mathrm{~mm}$ in extent along any axis. The only part of the PI not labeled in our experiments was its ventroposterior portion. However, this region is known to represent superior visual fields (Benevento and Rezak, 1976; Bender, 1981), and our injections were confined to the representation of lower fields and the horizontal meridian in MT. Our results are thus consistent with the possibility that MT has connections with the entire PI. Moreover, the label we found in the PL was restricted to approximately that portion which, along with the PI, makes up a single representation-the "inferior map" 


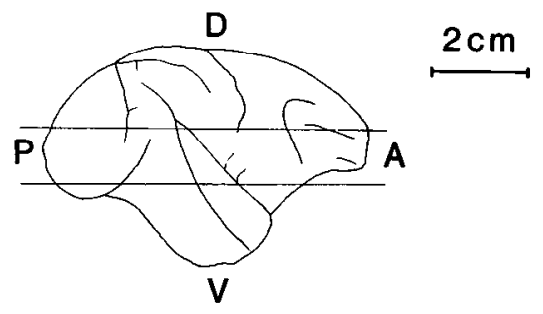

A

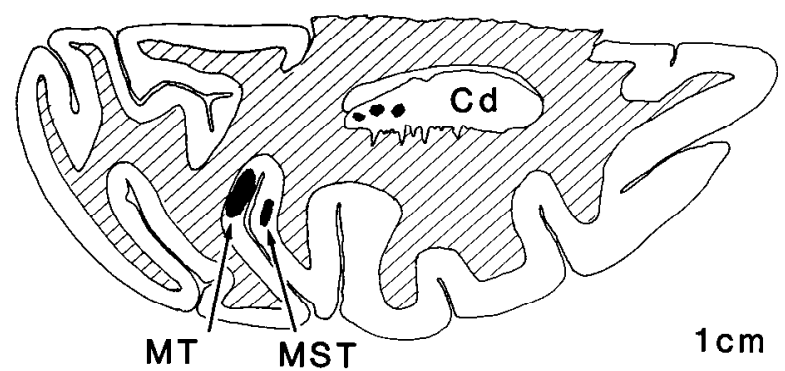

B

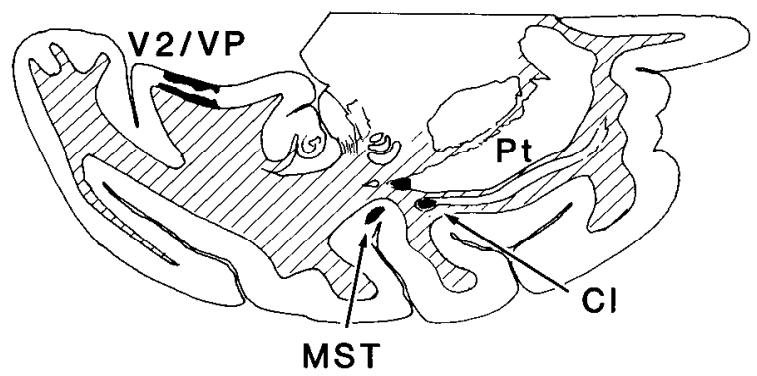

Figure 11. Labeling of the claustrum and basal ganglia following an injection of MT. An inset shows the level of two horizontal sections from the hemisphere of injection $3 . A$, The projection to the caudate nucleus. The caudate nucleus $(C d)$ contained small patches of anterogradely transported label over an extent of $7 \mathrm{~mm}$. $B$, The projections to the claustrum and putamen. Several small patches of anterogradely transported label were found in the claustrum $(\mathrm{Cl})$ for about $5 \mathrm{~mm}$ along its posterior margin. There was very faint radioactive label in the putamen $(P t)$ close to its posterior limit at this level. There was also label in several cortical visual areas at this level.

(Bender, 1981). We did not find the labeled regions in the pulvinar complex to be elongated along the rostrocaudal axis, however, as would be expected for the lines of projection described by Bender (1981).

It is difficult to reconcile our results with the suggestion of a narrow $\mathrm{MT}$ projection crescent in the pulvinar complex (Standage and Benevento, 1983). It may be that their use of standardized section outlines for displaying results from all of the injections contributed to an underestimate of the full extent of MT projections. In any event, additional experiments will be needed to resolve these issues definitively.

The thalamic reticular nucleus also receives a projection from MT. Axon terminations were found mainly in the region where this nucleus is pierced by labeled axons heading to the pulvinar complex. This type of labeling has been seen for cortical projections to the reticular nucleus in other primates (Spatz and Tigges, 1973; Lin and Kaas, 1977; Wall et al., 1982). The third diencephalic target of MT is the caudal subdivision of the pregeniculate nucleus (Olszewski, 1952), which is the portion lacking a direct retinal input (Hendrickson et al., 1970). The dorsal lateral geniculate nucleus (dLGN) was carefully examined for label, but none was found in any case.

Mesencephalon and pons. There is a moderate projection from MT to the ipsilateral superior colliculus. Label in the colliculus was confirmed to a single patch about $1.5 \mathrm{~mm}$ in diameter and was primarily in the stratum griseum superficiale, which also receives direct input from the retina and other cortical visual areas (see Rodieck, 1979). The position of the label within the superior colliculus was different in each case and corresponded well with the known topography (Cynader and Berman, 1972).

MT also sends a strong projection to the ipsilateral pontine nuclei. Radioactive label was seen in a rostrocaudally oriented column about $500 \mu \mathrm{m}$ in diameter and $4 \mathrm{~mm}$ in length. The label was not continuous within this column but was broken into irregular clumps. Labeled cells are also found in clumps in the pontine nuclei following HRP injections of the cerebellum (Brodal, 1982). Rostrally, the label was in the lateral part of the nucleus peduncularis and, caudally, it was near the border between the nucleus lateralis and the nucleus dorsolateralis (Nyby and Jansen, 1951). This region of the pontine nuclei is known to receive cortical input primarily from visual cortex (Brodal, 1978), and it is this same part of the pons that receives a projection from MT in other primates (Spatz and Tigges, 1973; Graham et al., 1979; Wall et al., 1982). Projections to this portion of the pons have previously been demonstrated by lesions (Glickstein et al., 1980) and $\left[{ }^{3} \mathrm{H}\right]$ proline injections (Fries, 1981) in the superior temporal sulcus in the macaque which, however, were not clearly restricted to MT. The response properties of visual neurons in this part of the pons of the monkey are not known, but in the cat (Baker et al., 1976), visually responsive cells in the pons are almost all direction selective and largely unselective for stimulus form, as are the neurons in macaque MT.

\section{Discussion}

Hierarchical organization of visual cortex. MT in the macaque has been shown to have a rich set of reciprocal connections with other areas in the visual cortex. On the basis of the laminar distributions of these connections, we have suggested that these areas consist of at least four and perhaps as many six which send forward projections to MT, two which receive forward projections from MT, and one which appears to be intermediate. The general notion that forward and feedback projections can be differentiated on the basis of laminar distributions has been presented in a number of earlier studies (Jones and Wise, 1977; Kaas et al., 1977; Tigges et al., 1977, 1981; Rockland and Pandya, 1979; Weller and Kaas, 1981). We propose here that this notion can be refined into an explicit hypothesis about hierarchical relationships among cortical visual areas in primates. 

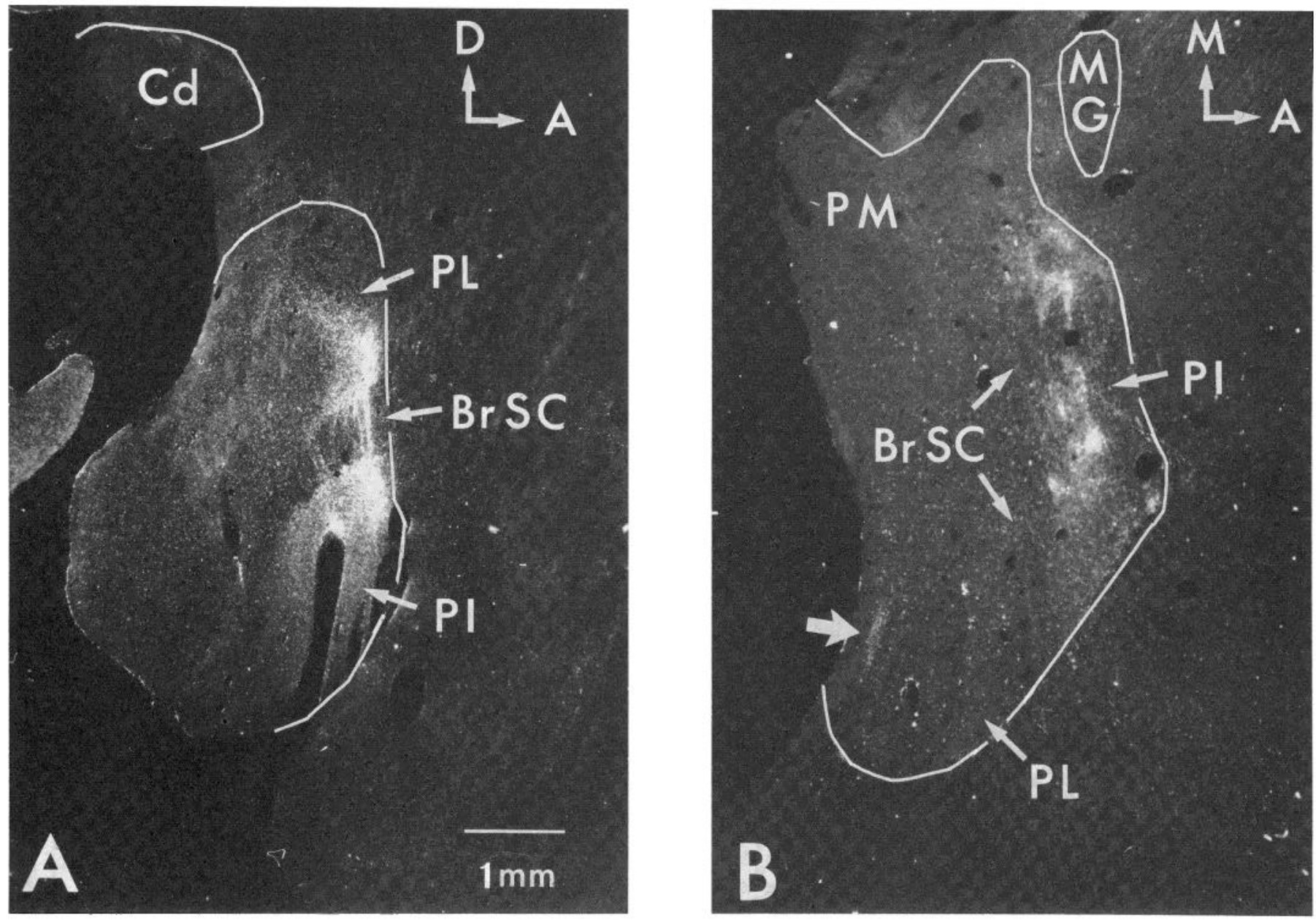

Figure 12. A, A darkfield photomicrograph of the projection from MT to the pulvinar complex. The section shown is from the hemisphere of injection 1, and the plane of sectioning is parasagittal. Clumps of label can be seen near the brachium of the superior colliculus $(B r S C)$ in both the inferior $(P I)$ and lateral $(P L)$ subdivisions of the pulvinar complex. $B$, A horizontal section through the hemisphere of injection 3 . The scale is identical to that in $A$. There are several patches of label in $P I$ and a single, faint patch in $P L$ (wide arrow). $C d$, caudate nucleus; $M G$, medial geniculate nucleus.

There are three major tenets of this hypothesis. (1) Connections between cortical areas are in general organized in reciprocal fashion. (2) With few exceptions, each reciprocal interconnection can be designated as forward-going in one direction and feedback in the other, based on characteristic differences in the laminar distribution of axonal terminations and cells of origin. We suggest that at least some of the exceptional cases are intermediate in their characteristics and symmetric for the two directions, and that they fit into a category of lateral interconnections. (3) These anatomical relationships can be used to delineate a well defined hierarchical order among all cortical visual areas.

The first point, that corticocortical connections in the visual system are reciprocal, is the most straightforward to discuss. All cortical projections of visual areas in the macaque are either known to be reciprocal or have not been adequately tested. The same is true of New World monkeys, with the exception that, in the owl monkey, the projection from the medial area (M) to V2 does not appear to be reciprocated (see Weller and Kaas, 1981; Tigges et al., 1981). In any event, occasional exceptions, even if confirmed using the most sensitive tracer techniques, do not pose a difficulty for the rest of the hypothesis.

The general appearance of the two types of projections under consideration is schematized in Figure 13. Forward projections, leading away from the primary cortical sensory areas, originate primarily or exclusively in supragranular layers and terminate for the most part in layer IV (specifically, the "granular" layer) and the lower part of layer III. Feedback projections, carrying information back toward the primary sensory areas, also originate to some extent in supragranular layers, but they include a substantial number of neurons, generally an outright majority, in the infragranular layers. Feedback projections terminate in supragranular and infragranular layers, often most densely in layers I and VI, thus avoiding the layers in which forward projections are concentrated.

Given that there is some variability in these laminar patterns, it is important to establish criteria for distinguishing between forward and feedback connections that are explicit enough to avoid ambiguities, yet not so restrictive as to lead to inappropriate assignments. There 


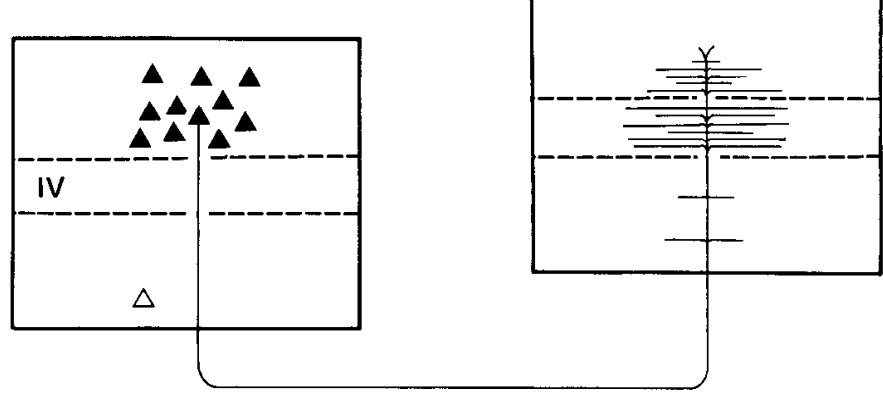

FORWARD

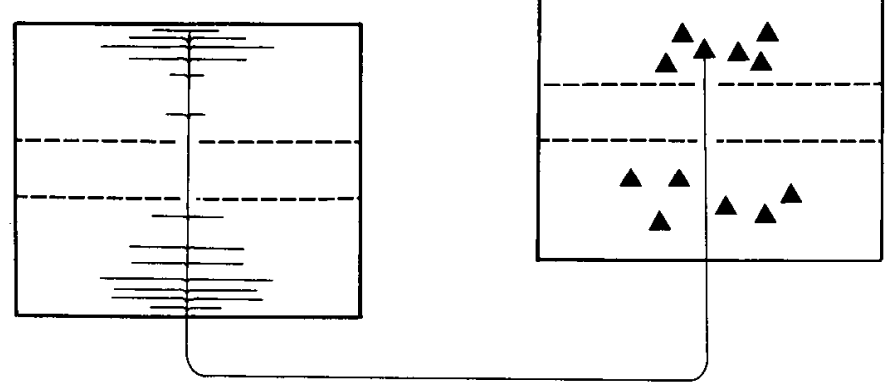

\section{FEEDBACK}

Figure 13. Characteristic laminar distributions of cell bodies and terminals in forward and feedback pathways. Forward pathways arise mainly from superficial layers and terminate mainly in layer IV. Feedback pathways arise from both superficial and deep layers and terminate mainly outside layer IV.

are several difficulties in achieving this, most of which can be illustrated by considering the example of the reciprocal connection between V1 and MT. The projection from V1 to MT differs from the prototypic forward projection in that it originates mainly in layer IVb (rather than layers II and III) and also in layer VI (which supposedly typifies feedback projections). The pathway from MT to V1 differs from the prototypic feedback projection in that it terminates mainly in a subdivision of layer IV, rather than in more superficial or deeper layers. Nonetheless, there are sound reasons for concluding that the appropriate polarity is indecd forward from V1 to MT and feedback from MT to V1. In relation to the layer IVb component, the key point is that this layer is more akin to the superficial layers (II and III) than to the other subdivisions of layer IV. It does not receive direct geniculate inputs (Hubel and Wiesel, 1972), and it projects to V2 as well as to MT (Maunsell et al., 1980; Tigges et al., 1981). This affinity of layer IVb with the superficial layers is reflected by its designation as a subdivision of layer III in some cortical layering schemes (Hassler, 1966; Spatz et al., 1970). In any event, these terminological difficulties can be avoided by referring to everything from layer IVb and above as supragranular, since layer IVc is the only true granular layer in V1. In relation to the layer VI component, the key issue is the magnitude of the projection relative to that arising from layer IVb. Our results indicate that only about $10 \%$ of the MT projection cells in V1 reside in layer VI. Comparably low estimates of the layer VI contribution can be inferred from the results of Spatz (1977) and Tigges et al. (1981) on New World monkeys. Along the same lines, occasional deep layer cells have been shown to contribute to the projection from V2 to MT (Tigges et al., 1981) and from V1 to V2 (Tigges et al., 198; our unpublished observations). Thus, the proposed scheme would break down altogether if the existence of a low percentage of infragranular projection cells were considered to rule out a forward projection. Fortunately, though, there is a much larger infragranular contribution to all of the putative feedback pathways under consideration (e.g., from MT to V1). Quantitative estimates are not available for most of these pathways, but the evidence from the present study as well as from other reports (Rockland and Pandya, 1979; Maunsell et al., 1980; Tigges et al., 1981) suggests that at least a third and often a majority of the cells contributing to feedback pathways are infragranular. On these grounds it appears

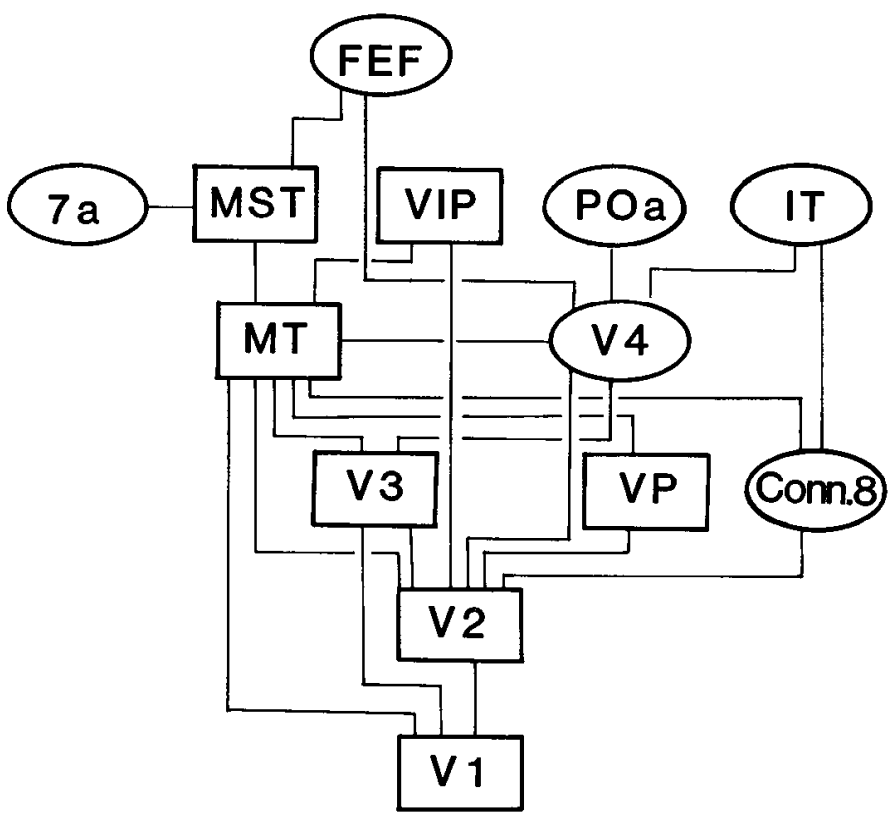

Figure 14. The hierarchy of cortical visual areas based on the laminar distributions of projections. Each visual area is assigned to the level immediately above the highest area from which it receives a forward projection. Rectangles indicate well established visual areas. This scheme is likely to be modified since there are undoubtedly more connections which have not yet been identified, and levels containing two or more areas may ultimately be split. Projections that are weak or inconsistent have not been included. A connection from MT to VP has been tentatively included (see "Results"). The connections shown and the laminar distributions used to assign levels in the hierarchy are from the present study and other investigations (see Table II). Some connections have not been established to be reciprocal; all those not as yet demonstrated are putative feedback projections (from V4 to V3 and V2, from VIP to V2, from IT to V4 and connection 8, and from POa to V4). 
TABLE II

Connections of macaque visual areas

\begin{tabular}{|c|c|c|c|c|c|}
\hline Pathway & Origin & Termination & Pathway & Origin & Termination \\
\hline $\mathrm{V} 1 \rightarrow \mathrm{V} 2$ & $7,11^{\alpha}$ & $3,11,16$ & $\mathrm{~V} 2 \rightarrow \mathrm{V} 1$ & $7,11,14,15$ & $7,11,15$ \\
\hline $\mathrm{V} 1 \rightarrow \mathrm{V} 3$ & & 3,16 & $\mathrm{~V} 3 \rightarrow \mathrm{V}_{1}$ & 13 & \\
\hline $\mathrm{V} 1 \rightarrow \mathrm{MT}$ & 6,8 & $3,11,14,16$ & $\mathrm{MT} \rightarrow \mathrm{V} 1$ & $11,14,15$ & 8 \\
\hline $\mathrm{V} 2 \rightarrow \mathrm{VP}$ & 2 & & $\mathrm{VP} \rightarrow \mathrm{V} 2$ & & 2 \\
\hline $\mathrm{V} 2 \rightarrow \mathrm{MT}$ & 8 & 11 & $\mathrm{MT} \rightarrow \mathrm{V} 2$ & 11 & 8 \\
\hline $\mathrm{V} 2 \rightarrow \mathrm{V} 4$ & & 11,17 & $\mathrm{~V} 4 \rightarrow \mathrm{V} 2$ & & 11 \\
\hline $\mathrm{V} 3 \rightarrow \mathrm{MT}$ & 9 & & $\mathrm{MT} \rightarrow \mathrm{V} 3$ & & 8 \\
\hline $\mathrm{V} 3 \rightarrow \mathrm{V} 4$ & & 17 & $\mathrm{~V} 4 \rightarrow \mathrm{V} 3$ & & \\
\hline $\mathrm{VP} \rightarrow \mathrm{MT}$ & & 2 & $\mathrm{MT} \rightarrow \mathrm{VP}$ & 2 & 8 \\
\hline $\mathrm{MT} \rightarrow \mathrm{V} 4$ & & 8 & $\mathrm{~V} 4 \rightarrow \mathrm{MT}$ & 8 & 11 \\
\hline $\mathrm{MT} \rightarrow \mathrm{MST}$ & & 8 & $\mathrm{MST} \rightarrow \mathrm{MT}$ & 8 & \\
\hline $\mathrm{MST} \rightarrow 7 \mathrm{a}$ & 9 & & $7 \mathrm{a} \rightarrow \mathrm{MST}$ & & 10 \\
\hline $\mathrm{MST} \rightarrow$ FEF & 1 & & FEF $\rightarrow$ MST & & 5 \\
\hline Conn. $8 \rightarrow$ MT & & & $\mathrm{MT} \rightarrow$ Conn. 8 & & 8 \\
\hline Conn. $8 \rightarrow$ IT & 4 & & IT $\rightarrow$ Conn. 8 & & \\
\hline
\end{tabular}

"The numbers indicate the source of information for each pathway, as follows: 1. Barbas and Mesulam, 1981; 2. Burkhalter, A. B., and D. C. Van Essen (unpublished); 3. Cragg, 1969; 4. Desimone et al., 1980; 5. Künzle and Akert, 1977; 6. Lund et al., 1976; 7. Maunsell et al., $1980 ; 8$. Maunsell and Van Essen (present study); 9. Mesulam et al., 1977; 10. Pandya and Seltzer, 1982; 11. Rockland and Pandya, 1979; 12. Seltzer and Pandya, 1980; 13. Van Essen, D. C., A. B. Burkhalter, W. T. Newsome, and J. H. R. Maunsell (unpublished); 14 . Weller and Kaas, $1978 ; 15$. Wong-Riley, 1979; 16. Zeki, 1969; 17. Zeki, 1971.

reasonable to regard an infragranular contribution of less than 10 to $15 \%$ as consistent with a forward pathway and a contribution of $30 \%$ or more as suggestive of a feedback pathway. An intermediate range should for the present be regarded as uncertain in assignment. As quantitative estimates become available for a larger number of pathways, these criteria for forward versus feedback patterns may, of course, be subject to revision.

Using the relationships between all pairs of reciprocally connected areas, it is possible to construct an overall hierarchical scheme for the ensemble of visual cortical areas. ${ }^{3}$ This can be done by assigning each area to a hierarchical level immediately above the uppermost level from which it receives a forward projection. The hierarchy for visual areas in the macaque is illustrated in Figure 14. The scheme involves six hierarchical levels occupied by seven well defined visual areas (boxed entries) and six less well defined visual regions (elliptical entries). The areas at the two uppermost levels of the hierarchy should not all be regarded as strictly visual in function. To the contrary, some of these are known either to have inputs from other sensory modalities or to be involved in visuomotor function (see Lynch, 1980; Goldberg and Bushnell, 1981).

This hierarchy is based on information from many

\footnotetext{
${ }^{3}$ By hierarchy, we mean a system in which each member can be unambiguously assigned to a specific level relative to other members. A serial ranking is one extreme form of hierarchy, but, of course, there are many other types. Note that a hierarchy need not have a single member at the top, nor decreasing numbers of members in successive levels.
}

different studies on cortical connections in the macaque. For every reciprocal pathway represented by one of the lines in Figure 14, there are four separate laminar patterns, relating to the origins and terminations of the projections in each direction. Table II provides a summary of which laminar patterns have been characterized, in a format that indicates the source of information for each pathway. It shows a total of 45 laminar patterns associated with 36 projections, including 14 pairs of identified reciprocal connections. Obviously, the list is far from complete, and determination of all four laminar patterns has been achieved for only three reciprocal pathways (V1-V2, V1-MT, and V2-MT).

It is of considerable significance that the hierarchy in Figure 14 can be constructed at all, since it depends on the fact that the connections noted in Table II adhere to two organizational relationships. The first has already been discussed; namely, that if one projection within a reciprocal pathway is of the forward type, the other is of the feedback type. The second is the principle of transitivity: if area $\mathrm{B}$ is forward relative to $\mathrm{A}$, and $\mathrm{C}$ is forward relative to $B$, then the projection from $A$ to $C$ (if it exists at all) is also forward. Clear exceptions to this principle would lead to a major ambiguity in the hierarchical assignments of the areas involved. On the other hand, it is not essential that all pathways conform to either the forward or feedback pattern. An obvious third possibility is that of "lateral" connections-between areas at the same hierarchical level. The existence of lateral connections does not invalidate the principle of hierarchical organization as long as any area which projects to two 
laterally connected areas bears the same relationship (forward, feedback, or lateral) to both.

It should not be surprising to find a few lateral connections in a hierarchy with as many members and interconnections as are already demonstrated in extrastriate visual cortex. The projection from MT to V4 may be one such example, since part of it terminates in a manner unlike either the standard forward or the feedback pattern, with label in radial bands extending through the thickness of the cortex. Rockland and Pandya (1979) described a similar pattern of columns in the superior temporal sulcus (probable MT) after tracer injections in "area 19" (probable V4), suggesting a reciprocal pathway which may be relatively symmetric. Radial columns are also a characteristic feature of some intrinsic cortical connections-those within a single area. This was shown by Rockland and Pandya (1979) for several extrastriate regions, and we have shown it in the present study for both the intrinsic ipsilateral connections within MT and also the contralateral projections to MT (but not MST). Intrinsic connections, be they ipsilateral or contralateral, link regions of cortex at the same hierarchical level and, thus, are a type of lateral connection. Accordingly, the similarity with other putative lateral connections may not be entirely fortuitous. We have also tentatively placed areas $7 \mathrm{a}$ and MST at the same hierarchical level, based on the limited information available concerning the pattern of connections between them (Künzle and Akert, 1977; Mesulam et al., 1977).

The hierarchical scheme we have discussed is based strictly on anatomical criteria and would have only limited utility if it were irrelevant to cortical function. In this regard, it is encouraging that there is a clear relationship between the physiological properties of cells in different areas and the position of these areas in the anatomically defined hierarchy. The strongest correlation is with receptive field size. Receptive fields at any given eccentricity are smallest in V1 and progressively increase in size at successive hierarchical levels (Gross et al., 1972; Robinson et al., 1978; Van Essen and Zeki, 1978; Gattass and Gross, 1981). The second type of correlation is with complexity of receptive field properties, which are simplest in V1 (Hubel and Wiesel, 1968) and most complex in the inferotemporal cortex and area 7a (Gross et al., 1972; Robinson et al., 1978; Mountcastle et al., 1981). This relationship is not as sharply defined, however, because not a great deal is known about differences in the complexity of receptive field organization at intermediate hierarchical levels.

As additional connections between visual areas are traced, it can be anticipated that the present scheme of hierarchical organization will be modified substantially by the incorporation of newly identified areas and perhaps also by splitting of what are currently regarded as single levels. Moreover, similar principles of hierarchical organization may also be demonstrable for other sensory modalities. If so, hierarchies for different sensory systems could meet at polysensory areas, such as those in the parietal cortex, and the cerebral cortex might be described as a single hierarchy of areas, albeit a highly complex one. On the other hand, it is possible that new findings could either invalidate the hierarchy altogether or limit its applicability to a portion of the visual pathway. In evaluating such possibilities, though, it is important to establish that the areas under consideration represent valid subdivisions of the cortex. For example, Brodmann's (1905) areas 18 and 19 in the macaque together contain seven of what are currently recognized as distinct visual areas on the basis of connections, topographic organization, and revised architectonic boundaries. Obviously, strict reliance on Brodmann's scheme for the analysis of connectional patterns in the occipital lobe would not have led to the recognition of a well defined hierarchy. Likewise, the assignment of hierarchical relationships for other cortical regions should be predicated upon a critical reassessment of the fundamental subdivisions of the parietal, temporal, and frontal lobes. Close attention to laminar patterns of connectivity may itself prove very helpful in refining the identification of cortical areas.

Another general issue concerns whether analogous hierarchies exist for species other than the macaque. We have already cited much of the evidence that the connections of visual areas in New World primates conform to the same laminar distribution patterns as in the macaque (cf. Weller and Kaas, 1981; Tigges et al., 1981). Hence, it seems likely that for a variety of primates there are cortical hierarchies which bear strong similarities to one another. In the cat, there is evidence based on retrograde labeling that there may be a clear distinction between forward and feedback pathways (Gilbert and Kelly, 1975). Less is known about differences in patterns of anterograde terminations between different areas. However, even if the criteria for distinguishing between forward and feedback pathways differ from those in primates, it is still possible that unambiguous hierarchical assignments could be made in the cat and other nonprimate species.

Little is known at present about the relationship between the cortical hierarchy and the extensive, often reciprocal, connections between cortical visual areas and various thalamic nuclei, especially the pulvinar. In the cat, Graybiel and Berson (1981) have suggested that visual cortical areas can be grouped into several "family clusters," based largely on the pattern of connections with the LGN and the lateralis posterior-pulvinar complex. It may turn out that similar groupings will become apparent for primates as more is learned about the precise patterns of connectivity between various subdivisions of the pulvinar complex and their targets in extrastriate visual cortex. If so, it will be of interest to see how such clusters are related to the hierarchy which has been identified in the present study strictly on the basis of corticocortical pathways.

The notion of hierarchical processing in visual cortex was first introduced by Hubel and Wiesel $(1962,1965)$ as a scheme to account for the receptive field properties of different cell types in areas 17, 18, and 19 of the cat. The emphasis in the present discussion has been on hierarchical relationships at a different level of organization, that of cortical areas rather than functional cell types. Of course, it would be very attractive if the hierarchy of areas could be refined and expanded to the extent that each area could be described in terms of an 
internal hierarchy of layers and/or cell types. On the other hand, there is no requirement that a system be hierarchical at every level of organization. For example, individual areas might be organized as complex networks lacking well defined levels of processing, while at the same time, the various areas considered as units could maintain precise hierarchical relationships to one another.

Topographic order, patchy projections, and extent of intrinsic connections. Several points should be considered in relation to the regional distribution (as distinct from laminar distribution) of connections within the various cortical areas to which MT projects. The first is simply that the connections with other topographically organized areas are restricted to regions representing the same part of the visual field as at the injection site. Although this is hardly a surprising result, it is significant in view of the substantial disorderliness of the visual representation within MT (Van Essen et al., 1981). Thus, the principle of linkage between topographically corresponding regions in different areas is upheld even when these regions are in different locations relative to areal boundaries (see Fig. 3, in which a vertical meridian injection links a site nearly midway across MT with portions of $\mathrm{V} 1$ and $\mathrm{V} 2$ right along their common border).

The second point about regional distribution is that the connections of MT are distinctly patchy in some areas, notably V2 and V4, but relatively uniform in others, especially V1. Patchy projections have now been described for a variety of intrahemispheric as well as interhemispheric pathways in primates (see Van Essen et al., 1982). Their significance is largely obscure, although in a general sense it is apparent that they may reflect a functional inhomogeneity within individual areas. With regard to the connections of MT, for example, one might expect that the patches of label in V2 and V4 would represent pockets of neurons whose physiological properties are appropriate for input to MT (i.e., selectivity for direction, disparity, and/or speed). An important question relating to this is whether or not the cortex in between the patches labeled by a focal tracer injection projects to any other regions within MT. This cannot be ascertained from our own experiments, but the results of Tigges et al. (1981) with large injections of HRP into MT suggest that there are labeled patches in V2 separated by cortex not projecting to MT at all. One might therefore expect to find significant differences in the properties of cells inside versus outside the MT projection patches.

The third point about regional distribution concerns the intrinsic connections of $\mathrm{MT}$, which are remarkedly extensive in relation to the overall dimensions of MT. In one instance (injection 3, Fig. 7) there was label across the full mediolateral extent of MT. In another (injection 1, Fig. 3), there was a patch of label located approximately $6 \mathrm{~mm}$ from the injection site. Because of their spread relative to visual field coordinates, these intrinsic connections are unlikely to be responsible for the main excitatory drive to neurons in MT. An interesting alternative is that they subserve a modulatory role in which a large surrounding region can influence the responsiveness to stimulation of the receptive field. Pronounced modulatory influences of this type, both facilitatory and inhibitory, have recently been shown to be common among neurons in MT of the owl monkey (Miezen et al., 1982). These surround effects sometimes extend over most of the visual field, which is commensurate with the physical extent of the intrinsic connections which we found for MT in the macaque and which Wagor et al. (1975) found in the owl monkey.

A related issue concerns the significance of the connections with MT of the opposite hemisphere. As already suggested, such interhemispheric projections are arguably an intrinsic connection of MT and, hence, they might serve the same modulatory role as the local ipsilateral connections just discussed. If so, one might expect that the contralateral projections originating from any given site in one hemisphere would be a continuation of the pattern found ipsilaterally, starting at the vertical meridian representation and extending, perhaps in a patchy fashion, a considerable distance away from this meridan.

An alternative possibility already mentioned is that interhemispheric connections preferentially link mirrorsymmetric portions of the visual field. Although the functional consequences seem potentially rather peculiar, evidence for such an arrangement has nonetheless been reported for several visual areas in monkeys and cats (Spatz and Tigges, 1972; Tigges et al., 1974; Wagor et al., 1975; Segraves and Rosenquist, 1982). However, in our opinion, this interpretation is open to doubt because the assessment of topographic relationships in these studies was based on standardized maps for one or both hemispheres rather than direct recording from the cortical regions in question. Our results, although not conclusive, argue against the extreme form of the mirrorimage hypothesis. Given the small size of the areas concerned and the substantial topographic disorderliness typical of extrastriate visual areas, though, it is apparent that further experiments involving detailed physiological as well as anatomical mapping of both contralateral and ipsilateral hemispheres will be necessary to resolve this issue.

Areas MST and VIP. An important result of these experiments has been the demonstration of a projection from MT to the cortex immediately medial to it in the superior temporal sulcus. This area is known to contain neurons which respond to visual stimuli and which have direction selectivity much like that in MT (Van Essen et al., 1981). We have called this the medial superior temporal area (MST) and define it as the MT-recipient region in the medial part of the superior temporal sulcus which contains a high proportion of direction-selective neurons. Newsome and Wurtz (1982) have recently described a distinctive architectonic region in the superior temporal sulcus, less heavily myelinated than MT, which may be coextensive with MST.

Previous anatomical and physiological experiments have demonstrated other features of MST. Labeled cells are found in MST following HRP injections in the frontal eye fields (Barbas and Mesulam, 1981) or area $7 \mathrm{a}(\mathrm{Me}-$ sulam et al., 1977). A study in alert monkeys by Newsome and Wurtz (1981) suggests that neurons in MST differ from those in MT in their responses to visual motions caused by eye movements. Whereas cells in MT respond 
similarly to equivalent retinal motions whether they are caused by stimulus or eye movements, some of the cells in MST respond during smooth eye movements in ways which are not explained by their responses to stimulus movements during stationary fixation. These anatomical and physiological results are consistent with the idea that MST represents a further stage in the processing of motion by the cortical visual system.

The ventral intraparietal area, VIP, like MST, is identified as a specific MT projection zone. It appears to be a small area, perhaps no larger than MT itself. Although it is included in the hierarchy of visual areas on the basis of its connections, we are not aware of any physiological evidence that this region, along the fundus of the intraparietal sulcus, actually contains visually responsive neurons. Nevertheless, these findings add to the evidence for considerable complexity in the anatomical organization of the posterior parietal cortex (cf. Pandya and Seltzer, 1982), thereby emphasizing the importance of relating the functional properties of cells to the particular parietal subdivision to which they belong.

Comparisons with other species. The connections of MT have been described in four other primate species: the owl monkey, squirrel monkey, marmoset, and bushbaby. MT in the owl monkey (Aotus) is known to have connections with five ipsilateral cortical areas (see Weller and Kaas, 1981). The connections of MT with V1 and $\mathrm{V} 2$ in this animal clearly correspond to those in the macaque. The dorsolateral area, which lies adjacent to MT in the owl monkey and which has connections with it, may be related to V4 in the macaque (Van Essen et al., 1982). The correlation between other MT-recipient areas in the owl monkey and the macaque is not clear.

The arrangement of cortical visual areas in the squirrel monkey (Saimiri), marmoset (Callithrix), and bushbaby (Galago) is less well understood, but MT in all three species has connections with V1 and V2 (Spatz and Tigges, 1972; Tigges et al., 1981; Wall et al., 1982). Some of the additional connections of MT in these species are likely to be equivalent to those seen in the macaque. MT in the bushbaby has contralateral projections to MT and to cortex dorsal to MT which might correspond to MST in the macaque (Wall et al., 1982). There are at least two differences between the cortical connections of $\mathrm{MT}$ in the macaque and the marmoset, though. Whereas MT in the marmoset appears to project to ventral V2, there is no projection further anterior in ventral cortex which can be equated to the projection from MT to connection 8 in the ventral cortex of the macaque. Furthermore, marmoset MT sends a substantial projection to the frontal eye fields. We found no such projection in the macaque.

For the most part, there is good agreement between the subcortical projections of MT in the macaque and other primate species, and these are summarized in Table III. We found no projection to the pretectum in the macaque. This projection is weak in the bushbaby (Wall et al., 1982), and it is possible that a small projection may have gone undetected in the macaque. In the owl monkey, MT has a weak projection to the magnocellular layers and layer S of the dLGN (Lin and Kaas, 1977; Graham et al., 1979). Although the dLGN was carefully
TABLE III

Subcortical targets of $M T$

\begin{tabular}{|c|c|c|c|c|}
\hline & Bushbaby $^{a}$ & Marmoset ${ }^{b}$ & Owl Monkey ${ }^{c}$ & Macaque $^{d}$ \\
\hline Claustrum & & + & & + \\
\hline Caudate nucleus & & + & + & + \\
\hline Putamen & + & + & + & + \\
\hline Reticular nucleus & + & + & + & + \\
\hline Pregeniculate nucleus & & + & $\begin{array}{l}+ \\
+\end{array}$ & + \\
\hline $\begin{array}{l}\text { dLGN } \\
\text { Pulvinar complex }\end{array}$ & + & + & $\begin{array}{l}+ \\
+\end{array}$ & + \\
\hline Lat. post. nucleus & + & & & \\
\hline Pretectum & + & + & + & \\
\hline Superior colliculus & + & + & + & + \\
\hline Pontine nucleus & + & + & + & + \\
\hline N. subthalamicus & & + & & \\
\hline $\begin{array}{l}\text { Nucleus ant. ophth. } \\
\text { tract }\end{array}$ & & + & & \\
\hline
\end{tabular}

${ }^{a}$ Wall et al., 1982; Raczkowski and Diamond, 1980, 1981.

${ }^{b}$ Spatz and Tigges, 1973.

${ }^{c}$ Graham et al. 1979; Lin and Kaas 1977, 1979.

${ }^{d}$ The present study.

examined, no such projection was seen in the macaque. Tigges et al. (1982) have recently shown that MT in the squirrel monkey (Saimiri) receives projections from at least 11 subcortical structures other than the pulvinar complex. None of these structures was demonstrated to project to MT in the present experiments. However, it is unlikely that the subcortical connections of MT are greatly different in the two species, and we presume that our experiments were not suited to demonstrate minor subcortical inputs (see "Materials and Methods").

Functional considerations of MT connections. The high proportion of neurons in MT which are selective for direction, speed, and disparity suggest that this area is important in the analysis of visual motion. The anatomical outputs of MT give further insight into how this type of information may be used in the nervous system.

MT projects to MST, which is also concerned with motion, and which in turn projects to area $7 \mathrm{a}$ (see above). Area 7 has been implicated in certain forms of complex behavior, including "attention" (Mountcastle et al., 1981). MT may provide an important sensory contribution to these functions. MT also projects to areas associated with the generation and control of eye movements, notably the superior colliculus and pontine nuclei. The superior colliculus in the macaque is known to contain a motor map of saccades (Schiller and Koerner, 1971). The MT-recipient part of the pons gives rise to a substantial projection to the part of the cerebellum around lobule VII (Brodal, 1978, 1982), which contains another motor map of eye movements (Ron and Robinson, 1973), and also neurons which signal eye speed and retinal slip speed (Suzuki et al., 1981).

As yet there is no evidence for a major input from MT to the inferotemporal cortex, aside from the relatively modest pathway from MT to V4, which then projects to IT. Inferotemporal cortex has been shown to contain neurons with a high degree of shape specificity (Gross et al., 1972) and is important for visual discrimination of form (see Gross, 1972). It would not be surprising if such connections were rather minor, since information from 
MT would be of little direct value for discrimination of form. The overall pattern of outputs from MT is consistent with the notion that extrastriate cortex contains relatively independent subsystems serving the analysis of motion and form discrimination. Further anatomical studies will be needed to determine how sharp this functional segregation actually is.

\section{References}

Baker, J., A. Gibson, M. Glickstein, and J. Stein (1976) Visual cells in the pontine nuclei of the cat. J. Physiol. (Lond.) 255: 415-433.

Barbas, H., and M. -M. Mesulam (1981) Organization of afferent input to subdivisions of area 8 in the rhesus monkey. $J$. Comp. Neurol. 200: 407-431.

Bender, D. B. (1981) Retinotopic organization of macaque pulvinar. J. Neurophysiol. 46: 672-693.

Benevento, L. A., and M. Rezak (1976) The cortical projections of the inferior pulvinar and adjacent lateral pulvinar in the rhesus monkey (Macaca mulatta): An autoradiographic study. Brain Res. 108: 1-24.

Brodal, P. (1978) The corticopontine projection in the rhesus monkey: Origin and principles of organization. Brain Res. 101: 251-283.

Brodal, P. (1982) Further observations on the cerebellar projections from the pontine nuclei and the nucleus reticularis tegmenti pontis in the rhesus monkey. J. Comp. Neurol. 204: $44-55$.

Brodmann, K. (1905) Beitrage zur histologische Localisation der Grosshirnrinde. Dritte Mitteilung. Die Rindenfelder der niederen Affen. J. Psychol. Neurol. 4: 177-226.

Cowan, W. M., D. L. Gottleib, A. E. Hendrickson, J. L. Price, and T. Woolsey (1972) The autoradiographic demonstration of axonal connections in the central nervous system. Brain Res. 37: 21-51.

Cragg, B. G. (1969) The topography of the afferent projections in the circumstriate visual cortex of the monkey studied by the Nauta method. Vision Res. 9: 733-747.

Cynader, M., and M. Berman (1972) Receptive field organization of monkey superior colliculus. J. Neurophysiol. 35: 187201.

Daniel, P. M., and D. Whitteridge (1961) The representation of the visual field on the cerebral cortex in monkeys. J. Physiol. (Lond.) 159: 203-221.

Desimone, R., J. Fleming, and C. G. Gross (1980) Prestriate afferents to inferior temporal cortex: An HRP study. Brain Res. 184: 41-55.

Fries, W. (1981) The projection from striate and prestriate visual cortex onto the pontine nuclei of the macaque monkey. Soc. Neurosci. Abstr. 7: 762.

Gallyas, F. (1979) Silver staining of myelin by means of physical development. Neurol. Res. 1: 203-209.

Gattass, R., and C. G. Gross (1981)Visual topography of striate projection zone (MT) in posterior superior temporal sulcus of the macaque. J. Neurophysiol. 46:621-638.

Gattass, R., C. G. Gross, and J. H. Sandell (1981) Visual topography of V2 in the macaque. J. Comp. Neurol. 201: 519539.

Gilbert, C. D., and J. P. Kelly (1975) The projection of cells in different layers of the cat's visual cortex. J. Comp. Neurol. 163: 81-106.

Glickstein, M., J. L. Cohen, B. Dixon, A. Gibson, M. Hollins, E. La Bossiere, and F. Robinson (1980) Corticopontine visual projections in macaque monkeys. J. Comp. Neurol. 190: 209229.

Goldberg, M. E., and C. Bushnell (1981) Behavioral enhancement of visual responses in monkey cerebral cortex. II. Mod- ulation in frontal eye fields specifically related to saccades. J. Neurophysiol. 46: 773-787.

Graham, J., C. S. Lin, and J. H. Kaas (1979) Sub-cortical projections of six visual cortical areas in the owl monkey, Aotus trivirgatus. J. Comp. Neurol. 187: 557-580.

Graybiel, A. M., and D. M. Berson (1981) On the relation between transthalamic and transcortical pathways in the visual system. In The Organization of the Cerebral Cortex, F. O. Schmitt, F. G. Worden, G. Adelman, and S. G. Dennis, eds., pp. 285-319, MIT Press, Cambridge, MA.

Grob, P., J. Büttner-Ennever, W. Lang, K. Akert, and A. Fäh (1982) A comparison of the retrograde tracer properties of $\left[{ }^{125}\right.$ I] wheat germ agglutinin with HRP after injection into the corpus callosum. Brain Res. 236: 193-198.

Gross, C. G. (1972) Visual functions of inferotemporal cortex. In Handbook of Sensory Physiology, R. Jung, ed., Vol. 7, Part 3 , pp. 451-482, Springer-Verlag, Berlin.

Gross, C. G., C. E. Rocha-Miranda, and D. B. Bender (1972) Visual properties of neurons in inferotemporal cortex of the monkey. J. Neurophysiol. 35: 96-111.

Hassler, R. (1966) Comparative anatomy of the central visual systems in day and night-active primates. In Evolution of the Forebrain, R. Hassler and H. Stephan, eds., pp. 419-434, Thieme Verlag, Stuttgart.

Hendrickson, A. E., M. E. Wilson, and M. J. Toyne (1970) The distribution of optic nerve fibers in Macaca mulatta. Brain Res. 23: 425-427.

Hubel, D. H., and T. N. Wiesel (1962) Receptive fields, binocular interaction and functional architecture in the cat's visual cortex. J. Physiol. (Lond.) 160: 106-154.

Hubel, D. H., and T. N. Wiesel (1965) Receptive fields and functional architecture in two non-striate areas (18 and 19) of the cat. J. Neurophysiol. 28: 229-289.

Hubel, D. H., and T. N. Wiesel (1968) Receptive fields and functional architecture of monkey striate cortex. J. Physiol. (Lond.) 195: 215-243.

Hubel, D. H., and T. N. Wiesel (1972) Laminar and columnar distribution of geniculo-cortical fibers in the monkey. J. Comp. Neurol. 146: 421-450.

Jones, E. G., and S. P. Wise (1977) Size, laminar and columnar distribution of efferent cells in the sensory motor cortex of monkeys. J. Comp. Neurol. 175: 391-438.

Jones, E. G., J. D. Coulter, and S. H. C. Hendry (1978) Intracortical connectivity of architectonic fields in the somatic sensory, motor, and parietal cortex of monkeys. J. Comp. Neurol. 181: 291-348.

Kaas, J. H., C. S. Lin, and E. Wagor (1977) Cortical projections of posterior parietal cortex in owl monkeys. J. Comp. Neurol. 171: 387-408.

Künzle, H., and K. Akert (1977) Efferent connections of cortical area 8 (frontal eye fields) in Macaca fascicularis. A reinvestigation using the autoradiographic technique. J. Comp. Neurol. 173: 147-164.

Lin, C. S., and J. H. Kaas (1977) Projections from cortical visual areas 17,18 , and $M T$ onto the dorsal lateral geniculate nucleus in owl monkeys. J. Comp. Neurol. 173: 457-474.

Lin, C. S., and J. H. Kaas (1979) The inferior pulvinar complex in owl monkeys: Architectonic subdivisions and patterns of input from the superior colliculus and subdivisions of visual cortex. J. Comp. Neurol. 187: 655-678.

Lin, C. S., and J. H. Kaas (1980) Projections from the medial nucleus of the inferior pulvinar complex to the middle temporal area of the visual cortex. Neuroscience 5: 2219-2228.

Lund, J. S., R. D. Lund, A. E. Hendrickson, A. H. Bunt, and A. F. Fuchs (1976) The origin of efferent pathways from the primary visual cortex, area 17 , of the macaque monkey as shown by retrograde transport of horseradish peroxidase. J. Comp. Neurol. 164: 287-304. 
Lynch, J. C. (1980) The functional organization of posterior parietal association cortex. Behav. Brain Sci. 3: 485-534.

Maunsell, J. H. R., and D. C. Van Essen (1982) The connections of the middle temporal visual area in the macaque monkey. Soc. Neurosci. Abstr. 8: 811.

Maunsell, J. H. R., and D. C. Van Essen (1983a) Functional properties of neurons in middle temporal visual area of the macaque monkey. I. Selectivity for stimulus direction, speed, and orientation. J. Neurophysiol. 49: 1127-1147.

Maunsell, J. H. R., and D. C. Van Essen (1983b) Functional properties of neurons in middle temporal visual area of the macaque monkey. II. Binocular interactions and sensitivity to binocular disparity. J. Neurophysiol. 49: 1148-1167.

Maunsell, .J. H. R., W. T. Newsome, and D. C. Van Essen (1980) The spatial organization of connections between V1 and V2 in the macaque: Patchy and non-patchy projections. Soc. Neurosci. Abstr. 6: 580.

Mesulam, M. -M. (1978) Tetramethyl benzidine for horseradish peroxidase neurohistochemistry: A non-carcinogenic blue reaction-product with superior sensitivity for visualizing neural afferents and efferents. J. Histochem. Cytochem. 26: 106117.

Mesulam, M. -M., G. W. Van Hoesen, D. N. Pandya, and N. Geschwind (1977) Limbic and sensory connections of the inferior lobule (area PG) in the rhesus monkey: A study with a new method for horseradish peroxidase histochemistry. Brain Res. 136: 393-414.

Miezen, F., E. McGuinness, and J. Allman (1982) Antagonistic direction-specific mechanisms in area MT in the owl monkey. Soc. Neurosci. Abstr. 8: 681.

Mountcastle, V. B., R. A. Andersen, and B. C. Motter (1981) The influence of attentive fixation upon the excitability of the light-sensitive neurons of the posterior parietal cortex. J. Neurosci. 1: 1218-1235.

Newsome, W. T., and R. H. Wurtz (1981) Response properties of single neurons in the middle temporal visual area (MT) of alert macaque monkeys. Soc. Neurosci. $\Lambda$ bstr. 7: 832.

Newsome, W. T., and R. H. Wurtz (1982) Identification of architectonic zones containing visual tracking cells in the superior temporal sulcus (STS) of macaque monkeys. Invest. Ophthalmol. Vis. Sci. 22: 238.

Newsome, W. T., J. H. R. Maunsell, and D. C. Van Essen (1980) Areal boundaries and topographic organization of the ventral posterior area (VP) of the macaque monkey. Soc. Neurosci. Abstr. 6: 579.

Nyby, O., and J. Jansen (1951) An experimental investigation of the corticopontine projection in Macaca mulatta. Norske Videnskaps-Academi. I. Matematisk-Naturviden Skapelag Klasse Skrifter 1-47.

Olszewski, J. (1952) The Thalamus of the Macaca mulatta, S. Karger AG, Basel.

Pandya, D. N., and B. Seltzer (1982) Intrinsic connections and architectonics of posterior parietal cortex in the rhesus monkey. J. Comp. Neurol. 204: 196-210.

Raczkowski, E., and I. T. Diamond (1980) Cortical connections of the pulvinar nucleus in Galago. J. Comp. Neurol. 193: 140.

Raczkowski, D., and I. T. Diamond (1981)Projections from the superior colliculus and the neocortex to the pulvinar nucleus in Galago. J. Comp. Neurol. 200: 231-254.

Robinson, D. L., M. E. Goldberg, and G. B. Stanton (1978) Parietal association cortex in the primate. Sensory mechanisms and behavioral modifications. J. Neurophysiol. 41: 910-932.

Rockland, K. S., and D. N. Pandya (1970) Laminar origins and terminations of cortical connection of the occipital lobe in the rhesus monkey. Brain Res. 179: 3-20.

Rodieck, R. W. (1979) Visual pathways. Annu. Rev. Neurosci.
2: $193-226$

Ron, S., and D. A. Robinson (1973) Eye movements evoked by cerebellar stimulation in the alert monkey. J. Neurophysiol. 36: 1004-1022.

Schein, S. J., R. T. Marrocco, and F. M. de Monasterio (1982) Is there a high concentration of color-selective cells in area V4 of monkey visual cortex? J. Neurophysiol. 47: 193-213.

Schiller, P. H., and F. Koerner (1971) Discharge characteristics of single units in superior colliculus of alert rhesus monkey. J. Neurophysiol. 34: 920-936.

Segraves, M. A., and A. C. Rosenquist (1982) The afferent and efferent callosal connections of retinotopically defined areas in cat cortex. J. Neurosci. 2: 1090-1107.

Seltzer, B., and D. N. Pandya (1980) Converging visual and somatic sensory cortical input to the intraparietal sulcus of the rhesus monkey. Brain Res. 192: 339-351.

Spatz, W. B. (1975) Thalamic and other subcortical projections to area MT (visual area of the superior temporal sulcus) in the marmoset Callithrix jacchus. Brain Res. 99: 129-134.

Spatz, W. B. (1977) Topographically organized reciprocal connections between area 17 and MT (visual area of superior temporal sulcus) in the marmoset Callithrix jacchus. Exp. Brain Res. 27: 559-572.

Spatz, W. B., and J. Tigges (1972) Experimental-anatomical studies on the "middle temporal visual area (MT)" in primates. I. Efferent cortico-cortical connections in the marmoset Callithrix jacchus. J. Comp. Neurol. 146: 451-464.

Spatz, W. B., and J. Tigges (1973) Studies on the visual area MT in primates. II. Projection fibers to subcortical structures. Brain Res. 61: 374-378.

Spatz, W. B., J. Tigges, and M. Tigges (1970) Subcortical projections, cortical associations, and some intrinsic interlaminar connections of the striate cortex in the squirrel monkey (Saimiri). J. Comp. Neurol. 140: 155-174.

Standage, G. P., and L. A. Benevento (1983) The organization of connections between the pulvinar and visual area MT in the macaque monkey. Brain Res. 262: 288-294.

Suzuki, D. A., H. Noda, and M. Kase (1981) Visual and pursuit eye movement-related activity in posterior vermis of monkey cerebellum. J. Neurophysiol. 46: 1120-1139.

Tigges, J., W. B. Spatz, and M. Tigges (1974) Efferent corticocortical fiber connection of area 18 in the squirrel monkey (Saimiri). J. Comp. Neurol. 158: 219-236.

Tigges, J., M. Tigges, and A. A. Perachio (1977) Complementary laminar terminations of afferents to area 17 orginating in area 18 and the LGN in squirrel monkey. J. Comp. Neurol. 176: 87-100.

Tigges, J., M. Tigges, S. Anschel, N. A. Cross, W. D. Letbetter, and R. L. McBride (1981) Areal and laminar distribution of neurons interconnecting the central visual cortical areas 17 , 18,19 , and MT in the squirrel monkey (Saimiri). J. Comp. Neurol. 202: 539-560.

Tigges, J., M. Tigges, N. A. Cross, R. L. McBride, W. D. Letbetter, and S. Anschel (1982) Subcortical structure projecting to visual cortical areas in squirrel monkey. J. Comp. Neurol. 209: 29-40.

Ungerleider, L. G., R. Desimone, and M. Mishkin (1982) Cortical projections of area MT in the macaque. Soc. Neurosci. Abstr. 8: 680.

Van Essen, D. C., and J. H. R. Maunsell (1980) Two-dimensional maps of cerebral cortex. J. Comp. Neurol. 191: 255281.

Van Essen, D. C., and S. M. Zeki (1978) The topographic organization of rhesus monkey prestriate cortex. J. Physiol. (Lond.) 277: 193-226.

Van Essen, D. C., J. H. R. Maunsell, and J. L. Bixby (1981) The middle temporal visual area in the macaque: Myeloarchitecture, connections, functional properties and topo- 
graphic organization. J. Comp. Neurol. 199: 293-326.

Van Essen, D. C., W. T. Newsome, and J. L. Bixby (1982) The pattern of interhemispheric connections and its relationship to extrastriate visual areas in the macaque monkey. J. Neurosci. 2: 265-283.

Wall, J. T., L. L. Symonds, and J. H. Kaas (1982) Cortical and subcortical projections of the middle temporal area (MT) and adjacent cortex in galagos. J. Comp. Neurol. 211: 193214.

Wagor, E., C. S. Lin, and J. H. Kaas (1975) Some cortical projections of the dorsomedial visual area (DM) of association cortex in the owl monkey, Aotus trivirgatus. J. Comp. Neurol. 163: 227-250.

Weller, R. E., and J. H. Kaas (1978) Connections of striate cortex with the posterior bank of the superior temporal sulcus in macaque monkeys. Soc. Neurosci. Abstr. 4: 650.

Weller, R. E., and J. H. Kaas (1981) Cortical and subcortical connections of the visual cortex in primates. In Cortical Sensory Areas. Vol. 2: Multiple Visual Areas, C. N. Woolsey, ed., pp. 121-155, Humana Press, Clifton, NJ.

Wiitanen, J. T. (1969) Selective silver impregnation of degenerating axons and axon terminals in the central nervous system of the monkey (Macaca mulatta). Brain Res. 14: 540-
548.

Wong-Riley, M. (1979) Columnar cortico-cortical interconnections within the visual system of the squirrel and macaque monkeys. Brain Res. 162: 201-217.

Zeki, S. M. (1969) Representation of central fields in prestriate cortex of monkey. Brain Res. 14: 271-291.

Zeki, S. M. (1970) Interhemispheric connections of prestriate cortex in monkey. Brain Res. 19: 63-65.

Zeki, S. M. (1971) Cortical projections from two prestriate areas in the monkey. Brain Res. 34: 19-35.

Zeki, S. M. (1974a) Functional organization of a visual area in the posterior bank of the superior temporal sulcus of the rhesus monkey. J. Physiol. (Lond.) 236: 549-573.

Zeki, S. M. (1974b) Cells responding to changing image size and disparity in the cortex of the rhesus monkey. J. Physiol. (Lond). 242: 827-841.

Zeki, S. M. (1977) Colour coding in the superior temporal sulcus of rhesus monkey visual cortex. Proc. R. Soc. Lond. Biol. 197: $195-223$.

Zeki, S. M. (1978) The cortical projections of foveal striate cortex in the rhesus monkey. J. Physiol. (Lond.) 277: 227244. 